\title{
The consistency strength of the tree property at the double successor of a measurable cardinal
}

by

\section{Natasha Dobrinen (Denver, CO) and Sy-David Friedman (Wien)}

Abstract. The Main Theorem is the equiconsistency of the following two statements:

(1) $\kappa$ is a measurable cardinal and the tree property holds at $\kappa^{++}$;

(2) $\kappa$ is a weakly compact hypermeasurable cardinal.

From the proof of the Main Theorem, two internal consistency results follow: If there is a weakly compact hypermeasurable cardinal and a measurable cardinal far enough above it, then there is an inner model in which there is a proper class of measurable cardinals, and in which the tree property holds at the double successor of each strongly inaccessible cardinal. If $0^{\#}$ exists, then we can construct an inner model in which the tree property holds at the double successor of each strongly inaccessible cardinal. We also find upper and lower bounds for the consistency strength of there being no special Aronszajn trees at the double successor of a measurable cardinal. The upper and lower bounds differ only by 1 in the Mitchell order.

1. Introduction. A fundamental theorem due to König states that every finitely branching tree with height $\omega$ contains an infinite branch [17]. In this paper, we are interested in when the analogous statement holds or fails for larger trees. We begin with some standard definitions which can be found in [13] and [16]. Let $\kappa$ be an infinite cardinal. A $\kappa$-tree is a tree $T$ of height $\kappa$ such that every level of $T$ has size less than $\kappa$. A tree $T$ is a $\kappa$-Aronszajn tree if $T$ is a $\kappa$-tree which has no cofinal branches. We say that the tree property holds at $\kappa$, or $\operatorname{TP}(\kappa)$ holds, if every $\kappa$-tree has a branch of length $\kappa$ through it. Thus, $\operatorname{TP}(\kappa)$ holds iff there is no $\kappa$-Aronszajn tree.

König's Lemma is equivalent to the statement $\mathrm{TP}\left(\aleph_{0}\right)$ holds. However, once we allow ourselves to consider uncountable cardinals $\kappa, \operatorname{TP}(\kappa)$ is not immediate from ZFC. In fact, Aronszajn showed in ZFC that there is an $\aleph_{1}$-Aronszajn tree (see [18]). (See also [13] for a proof.) Hence, $\operatorname{TP}\left(\aleph_{1}\right)$ fails in ZFC. Moreover, Specker proved that whenever $\kappa^{<\kappa}=\kappa$, then there is a 
$\kappa^{+}$-Aronszajn tree [26]. It follows that, for an infinite cardinal $\kappa$, if $\operatorname{TP}\left(\kappa^{++}\right)$ holds, then $2^{\kappa}$ must be at least $\kappa^{++}$. Jensen showed that a weak form of $\square_{\kappa}$ is equivalent to the existence of a special Aronszajn tree on $\kappa^{+}$[14].

Large cardinals are often needed once we consider trees of height greater than $\aleph_{1}$. Silver proved in [25] that for $\kappa>\aleph_{1}, \operatorname{TP}(\kappa)$ implies $\kappa$ is weakly compact in $L$. Mitchell proved in [21] that given a weakly compact cardinal $\lambda$ above a regular uncountable cardinal $\kappa$, one can turn $\lambda$ into $\kappa^{+}$so that in the extension model, $\kappa^{+}$has the tree property. Thus, $\operatorname{TP}\left(\aleph_{2}\right)$ is equiconsistent with the existence of a weakly compact cardinal.

It became of interest to see whether there were simpler methods of forcing the tree property to hold. Baumgartner and Laver simplified Mitchell's proof in [2] by using instead a weakly compact length countable support iteration of Sacks forcing to obtain the tree property at $\aleph_{2}$ in a generic extension. This generalizes to the following: If $\rho$ is strongly inaccessible and $\lambda>\rho$ is weakly compact, then iterated $\operatorname{Sacks}(\rho)$ forcing of length $\lambda$ with $\rho$ size supports yields $\operatorname{TP}\left(\rho^{++}\right)$in the generic extension [15]. (See the definitions in Section 2 and Theorem 3.2.) More generally, Kanamori proved that for any regular cardinal $\kappa$, assuming $\nabla_{\kappa}$, iterated $\operatorname{Sacks}(\kappa)$ forcing of weakly compact length gives $\operatorname{TP}\left(\kappa^{++}\right)$. Kanamori claims (but does not explicitly prove) that when $\kappa$ is strongly inaccessible then $\nabla_{\kappa}$ is not necessary; so we include a proof of this in Section 3. In fact, Baumgartner showed that the countable support iteration of many other forcings (including $\omega$-Cohen forcing) of weakly compact length produces models of the tree property at $\aleph_{2}$ (see [22]).

For more of the relevant literature on the tree property, we refer the reader to the following. Abraham [1], Cummings and Foreman [3], and Foreman, Magidor and Schindler [5] have done work on the tree property at two or more successive cardinals. Magidor and Shelah [20] have worked on the tree property at successors of singular cardinals. Schindler [24] has results on the tree property and weak covering.

In this paper we are interested in the consistency strength of the tree property at the double successor of a measurable cardinal. By results of Gitik [11], it is necessary to have $\mathrm{o}^{K}(\kappa) \geq \kappa^{++}$(in the Mitchell order for measures) in order to have GCH fail at $\kappa$, where $\kappa$ is a measurable cardinal. It is also known that a supercompact cardinal $\kappa$ with a weakly compact cardinal above suffices to obtain a model in which $\kappa$ is still supercompact and $\operatorname{TP}\left(\kappa^{++}\right)$ holds; after a Laver preparation for preservation of the supercompact by $\kappa$-directed closed forcings (see [19]), forcing with a weakly compact length iteration of $\operatorname{Sacks}(\kappa)$ forcings gives $\operatorname{TP}\left(\kappa^{++}\right)$.

DeFinition 1.1. Let $\kappa$ be a strongly inaccessible cardinal. We say that $\kappa$ is weakly compact hypermeasurable if there is a weakly compact cardinal 
$\lambda>\kappa$ and an elementary embedding $j: V \rightarrow M$ such that $\kappa=\operatorname{crit}(j)$ and $(H(\lambda))^{V}=(H(\lambda))^{M}$.

The main theorem of this paper is the following, the consistency being shown in Theorem 4.1, the necessity of a weakly compact hypermeasurable being shown in Theorem 5.3 .

Main Theorem. " $\kappa$ is a measurable cardinal and $T P\left(\kappa^{++}\right)$holds" is equiconsistent over ZFC with " $\kappa$ is weakly compact hypermeasurable."

From the proof of the Main Theorem, two internal consistency results follow:

THEOREM 6.1. If $V \models G C H$ and has a weakly compact hypermeasurable cardinal $\kappa$ and a measurable cardinal $\mu$ sufficiently large above $\kappa$, then there is an inner model of $V$ in which there is a proper class of measurable cardinals, and in which the tree property holds at the double successor of each strongly inaccessible cardinal.

TheOREM 6.2. Suppose $0^{\#}$ exists. Then there is an inner model in which the tree property holds at the double successor of every strongly inaccessible cardinal.

The paper is organized as follows. Section 2 contains the necessary definitions and facts. In Section 3, since a self-contained proof has not been available in the literature, we provide a proof that the tree property at the double successor of a strongly inaccessible cardinal can be obtained from iterated Sacks forcing, assuming the existence of a weakly compact cardinal above the strongly inaccessible. The heart of the paper is Section 4, where we show that we can preserve the measurability of a weakly compact hypermeasurable cardinal while obtaining the tree property at the double successor of the measurable. Here, we perform a reverse Easton iteration of iterated Sacks forcings. In order to show that measurability is preserved, we use ideas from the "Tuning Fork" method of Friedman and Thompson in [10], which gave a more streamlined proof of a result of Woodin regarding failure of GCH at a measurable cardinal. However, new challenges arise due to the fact that we are not using a Sacks product as in [10, but rather an iteration. These difficulties are addressed in Lemmas 4.7 and 4.9 , and also in Lemmas 2.8 and 2.9. In Section 5 we generalize Silver's proof that for uncountable $\kappa$, $\mathrm{TP}(\kappa)$ implies $\kappa$ is weakly compact in $L$. Under some additional hypotheses, we construct a Silver-type tree in the core model $K$ instead of $L$, allowing us to obtain the lower bound on the consistency strength of the tree property at the double successor of a measurable. Putting together the work from Sections 4 and 5 yields the Main Theorem. From the proof of the Main Theorem, we deduce in Section 6 Theorems 6.1 and 6.2 regarding the internal consistency of the tree property. We conclude this paper in Section 7 with 
upper and lower bounds (with a gap only of length 1 in the Mitchell order) of the consistency strength of the statement " $\kappa$ is measurable and there are no special $\kappa^{++}$-Aronszajn trees."

The referee is to be thanked for a very careful reading of this paper which resulted in the fixing of many typos and many details being added. The referee's help was also valuable in catching an important oversight in our initial proof of Theorem 4.1 and which we overcame by the use of an iteration of sum forcings (see the proof of Theorem 4.1 for more details).

2. Definitions and Lemmas. The concept of a weakly compact cardinal has arisen independently in different areas of logic; hence there are many different formulations of weak compactness.

Definition 2.1 ([16]). Let $\kappa$ be a regular uncountable cardinal. We say that $\kappa$ is weakly compact if any collection of $L_{\kappa \kappa}$ sentences using at most $\kappa$ non-logical symbols, if $\kappa$-satisfiable, is satisfiable.

The following theorem lists several equivalences of weak compactness, all (except for (3)) of which appear in [16].

THEOREM 2.2. Let $\kappa$ be an uncountable regular cardinal. The following are equivalent:

(1) $\kappa$ is weakly compact.

(2) $\kappa$ is strongly inaccessible and $T P(\kappa)$.

(3) $\kappa$ is strongly inaccessible and for every transitive model $M$ of $Z F^{-}$ such that $\kappa \in M, M$ is $\kappa$-closed and $|M|=\kappa$, there is an elementary embedding $j: M \rightarrow N, N$ transitive, with $\operatorname{crit}(j)=\kappa$.

(4) $\kappa \rightarrow(\kappa)^{2}$.

The first investigation of Sacks forcing was in [23]. Baumgartner and Laver were the first to use Sacks forcing to obtain the tree property at $\aleph_{2}$ in [2]. Subsequent work augmenting their results for regular uncountable cardinals appears in Kanamori [15.

We now define Sacks forcing on $2^{<\rho}$ for $\rho$ a strongly inaccessible cardinal and give some basic definitions and facts. For a strongly inaccessible cardinal $\rho$, our version of $\operatorname{Sacks}(\rho)$ forcing forms a dense subset of the version of Sacks $(\rho)$ which Kanamori gives in [15]; hence, they are forcing equivalent. Our version helps to simplify notation throughout the paper. Definitions 2.4 and 2.5 come from [15], though their first versions related specifically to the tree property at $\aleph_{2}$ have precedents in [2]. Fact 2.6 and the Fusion Method Using Canonical Names are, as far as we know, new to this paper, though the general idea of Fact 2.6 is folklore. Fact 2.7 has a precedent in [2] and is stated in [15]. Lemma 2.8 is new to this paper, though the analogue of it for a product of Sacks forcings is found in [10. Lemma 2.9 is new to this paper. 
Definition 2.3. For strongly inaccessible $\rho$, let $\operatorname{Sacks}(\rho)$ denote the following forcing. A condition is a subset $p$ of $2^{<\rho}$ such that

(1) $s \in p, t \subseteq s \rightarrow t \in p$.

(2) Each $s \in p$ has a proper extension in $p$.

(3) For any $\alpha<\rho$, if $\left\langle s_{\beta}: \beta<\alpha\right\rangle$ is a sequence of elements of $p$ such that $\beta<\beta^{\prime}<\alpha \rightarrow s_{\beta} \subseteq s_{\beta^{\prime}}$, then $\bigcup\left\{s_{\beta}: \beta<\alpha\right\} \in p$.

(4) Let $\operatorname{Split}(p)$ denote the set of $s \in p$ such that both $s \frown 0$ and $s \frown 1$ are in $p$. Then for some (unique) club denoted $C(p) \subseteq \rho, \operatorname{Split}(p)=\{s \in$ $p: \operatorname{length}(s) \in C(p)\}$.

Extension is defined by $q \leq p$ iff $q \subseteq p$, where $q \leq p$ means that $q$ is stronger than $p$.

We take this opportunity to note that if the meaning is clear from the context, we shall often leave off checks and dots from above names in the forcing language so that the notation is less cumbersome.

Sacks $(\rho)$ is a $\rho$-closed forcing. (A forcing $\mathbb{P}$ is $\rho$-closed if for each $\alpha<\rho$, every decreasing sequence $\left\langle p_{\beta}: \beta<\alpha\right\rangle$ of elements in $\mathbb{P}$ has a lower bound in $\mathbb{P}$.) If $2^{\rho}=\rho^{+}$, then $|\operatorname{Sacks}(\rho)|=\rho^{+}$; hence, $\operatorname{Sacks}(\rho)$ has the $\rho^{++}$-c.c. Sacks $(\rho)$ preserves $\rho^{+}$, since it obeys the following $\rho$-fusion property. Given $p \in \operatorname{Sacks}(\rho)$, let $\left\langle\gamma_{\alpha}: \alpha<\rho\right\rangle$ be the increasing enumeration of $C(p)$. For $\alpha<\rho$, the $\alpha$ th splitting level of $p$, $\operatorname{Split}_{\alpha}(p)$, is the set of $s \in p$ of length $\gamma_{\alpha}$. For $\alpha<\rho$ we write $q \leq_{\alpha} p$ iff $q \leq p$ and $\operatorname{Split}_{\alpha}(q)=\operatorname{Split}_{\alpha}(p)$.

$\rho$-fusion: Suppose $\left\langle p_{\alpha}: \alpha<\rho\right\rangle$ is a sequence of elements of $\operatorname{Sacks}(\rho)$ such that for each $\beta<\alpha<\rho, p_{\alpha} \leq_{\beta} p_{\beta}$. Then $\bigcap_{\alpha<\rho} p_{\alpha} \in \operatorname{Sacks}(\rho)$.

$\rho$-fusion implies that $\rho^{+}$is preserved. For $p \in \operatorname{Sacks}(\rho)$ and a node $t \in p$, let $(p)_{t}$ denote $\{s \in p: s \leq t$ or $s \geq t\}$. Suppose we are given $p_{0} \in \operatorname{Sacks}(\rho)$ and a name for a function $\dot{f}: \rho \rightarrow \rho^{+}$. For $\alpha<\rho$, given $p_{\alpha}$, let $T_{\alpha}$ denote the collection of all immediate successors of nodes in $\operatorname{Split}_{\alpha}\left(p_{\alpha}\right)$. For each $t \in T_{\alpha}$, take a $q_{\alpha, t}^{\prime} \leq\left(p_{\alpha}\right)_{t}$ which decides the value of $\dot{f}(\alpha)$. Let $C_{\alpha}=\bigcap_{t \in T_{\alpha}} C\left(q_{\alpha, t}^{\prime}\right)$. Since $\rho$ is inaccessible and $\left|T_{\alpha}\right|=2^{\alpha+1}<\rho, C_{\alpha}$ is again a club subset of $\rho$. Thin each $q_{\alpha, t}^{\prime}$ to some $q_{\alpha, t} \leq q_{\alpha, t}^{\prime}$ such that $C\left(q_{\alpha, t}\right)=C_{\alpha}$. Now let $p_{\alpha+1}=\bigcup_{t \in T_{\alpha}} q_{\alpha, t}$. Note that $p_{\alpha+1} \leq_{\alpha} p_{\alpha}$. For limit ordinals $\alpha<\rho$, given $p_{\beta}$ for all $\beta<\alpha$, let $p_{\alpha}=\bigcap_{\beta<\alpha} p_{\beta}$. Then $p_{\alpha} \leq_{\beta} p_{\beta}$ for all $\beta<\alpha<\rho$. Let $p_{*}=\bigcap_{\alpha<\rho} p_{\alpha}$. Note that for each $\alpha<\rho, p_{\alpha}$ forces $\dot{f}(\alpha)$ to belong to a subset of $\rho^{+}$of size at most $\left|2^{\alpha+1}\right|<\rho$, since $\rho$ is inaccessible. Thus, there is a set $F$ of cardinality $\rho$ such that for each $\alpha<\rho, p_{*}$ forces $\dot{f}(\alpha)$ to be in $F$. Hence, $p$ forces that $\dot{f}$ is not onto $\rho^{+}$.

Although the following definitions, facts, and lemmas may be made more general, for the purposes of this paper it suffices to consider iterations of length $\lambda$, a weakly compact cardinal above $\rho$. 
Definition 2.4. Let $\rho<\lambda$ be cardinals with $\rho=\omega$ or $\rho$ strongly inaccessible, and $\lambda$ weakly compact. Let $\operatorname{Sacks}(\rho, \lambda)$ denote the $\lambda$-length iteration of $\operatorname{Sacks}(\rho)$ with supports of size $\leq \rho$. That is, let $\mathbb{S}_{0}=\{\emptyset\}$. For $i<\lambda$, let $\dot{\mathbb{R}}_{i}$ be an $\mathbb{S}_{i}$ name such that $\mathbb{S}_{i} \Vdash \dot{\mathbb{R}}_{i}$ is $\operatorname{Sacks}(\rho)$. $\operatorname{Sacks}(\rho, \lambda)$ is the iteration $\left\langle\left\langle\mathbb{S}_{i}, \dot{\mathbb{R}}_{i}\right\rangle: i<\lambda\right\rangle$ with supports of size at most $\rho$. For $p \in \mathbb{S}$ and $i<\lambda$, we let $p \nmid i$ denote the portion of $p$ in $\mathbb{S}_{i}$; that is, $p$ restricted to indices below $i$. For $i<i^{\prime} \leq \lambda$, we let $p\left\lceil\left[i, i^{\prime}\right)\right.$ denote the $\mathbb{S}_{i}$ name for the portion of $p$ on indices $k \in\left[i, i^{\prime}\right)$. When there is no confusion about which cardinals $\rho, \lambda$ are being used, we simply let $\mathbb{S}$ denote $\operatorname{Sacks}(\rho, \lambda)$.

Definition 2.5 (Generalized $\rho$-fusion). For $\alpha<\rho, X \subseteq \lambda$ of size less than $\rho$, and $p, q \in \operatorname{Sacks}(\rho, \lambda)$, we write $q \leq_{\alpha, X} p$ iff $q \leq p$ (i.e., $q\lceil i \Vdash q(i) \leq$ $p(i)$ for each $i<\lambda)$ and in addition, for each $i \in X, q\left\lceil i \Vdash q(i) \leq_{\alpha} p(i)\right.$.

Suppose that $\left\langle p_{\alpha}: \alpha<\rho\right\rangle$ is a decreasing sequence in $\operatorname{Sacks}(\rho, \lambda)$ such that $p_{\alpha} \leq_{\beta, X_{\beta}} p_{\beta}$ for each $\beta<\alpha<\rho$, where the $X_{\alpha}$ 's form an increasing sequence of subsets of $\lambda$ each of size less than $\rho$ whose union is the union of the supports of the $p_{\alpha}$ 's. Then the $p_{\alpha}$ 's have a lower bound in $\operatorname{Sacks}(\rho, \lambda)$; namely $q$, where $q(0)=\bigcap_{\alpha<\rho} p_{\alpha}(0), q(1)$ is an $\mathbb{S}_{0}$ name such that $q(0) \Vdash$ $q(1)=\bigcap_{\alpha<\rho} p_{\alpha}(1)$; in general for $i<\lambda, q(i)$ is an $\mathbb{S}_{i}$ name such that $q\left\lceil i \Vdash q(i)=\bigcap_{\alpha<\rho} p_{\alpha}(i)\right.$. Moreover, for each $\alpha<\rho, q \leq_{\alpha, X_{\alpha}} p_{\alpha}$.

When working with an iteration, the partial ordering is usually truly only a quasi-order, not being anti-symmetric. Suppose $\mathbb{P}=\left\langle\left\langle\mathbb{P}_{i}, \dot{\mathbb{Q}}_{i}\right\rangle: i<\theta\right\rangle$ is some forcing iteration. For $p, q \in \mathbb{P}$, we write $p \sim q$ iff $p \leq q$ and $q \leq p$.

Let $\tilde{D}$ denote the set of conditions $p \in \mathbb{P}$ such that for each $i<\theta$, $\mathbb{P}_{i} \Vdash p(i) \in \dot{\mathbb{Q}}_{i}$. Moreover, $\operatorname{supp}(p)$ denotes $\left\{i<\kappa: p \nmid i \Downarrow p(i)=\mathbf{1}_{i}\right\}$, the support of $p$, and length $(p)$ denotes $\sup \{i+1: i \in \operatorname{supp}(p)\}$.

FACT 2.6. Suppose $\mathbb{P}=\left\langle\left\langle\mathbb{P}_{i}, \dot{\mathbb{Q}}_{i}\right\rangle: i<\theta\right\rangle$ is some forcing iteration, and let $\tilde{D}$ denote the set of conditions $p \in \mathbb{P}$ such that for each $i<\theta$, $\mathbb{P}_{i} \Vdash p(i) \in \dot{\mathbb{Q}}_{i}$. If $q \leq p$ and $p \in \tilde{D}$, then there is an $r \sim q$ (i.e. $r \leq q$ and $q \leq r)$ such that $r \in \tilde{D}$ and for all $i<\theta, \mathbb{P}_{i} \Vdash r(i) \leq p(i)$.

Hence, we will work inside the dense subset $\tilde{D}$ of any iterated forcing occurring in this paper.

There are different ways one could construct a fusion sequence in iterated Sacks forcing. Here, we give a concrete method, which suffices for many (but not all) of the fusion arguments needed in this paper. (See Definitions 4.6 and 4.8 and Lemma 4.7 of Section 4 for a different method for obtaining fusion sequences, using conditions with certain portions being "determined" in the ground model.) Whenever one uses the following method for constructing a fusion sequence, one can practically think of the iteration as a product.

Fusion Method Using Canonical Names. Let $p \in \operatorname{Sacks}(\rho, \lambda)$. Without loss of generality, by Fact 2.6 we can assume $p \in \tilde{D}$. The following 
is a general means for constructing a fusion sequence $\left\langle q_{\alpha}: \alpha<\rho\right\rangle$ below $p$. Let $q_{0}=p$. Given $\alpha<\rho$ and $q_{\alpha} \in \tilde{D}$, if $\alpha$ is a successor ordinal, take $X_{\alpha} \subseteq \operatorname{supp}\left(p_{\alpha-1}\right)$ such that $\left|X_{\alpha}\right|<\rho$; if $\alpha$ is a limit ordinal, let $X_{\alpha}=\bigcup_{\beta<\alpha} X_{\beta}$. For each $i \in X_{\alpha}$ and $\zeta \in 2^{\alpha}$, let $\dot{s}\left(q_{\alpha}, i, \alpha, \zeta\right)$ be a $\operatorname{Sacks}(\rho, \lambda)\left\lceil i\right.$ name for the $\zeta$ th element in $\operatorname{Split}_{\alpha}\left(q_{\alpha}(i)\right.$ ) (under the natural bijection between $\operatorname{Split}_{\alpha}\left(q_{\alpha}(i)\right)$ and $\left.2^{\alpha}\right)$. Let $\tilde{r}^{m}(i, \alpha, \zeta)$ be any $\operatorname{Sacks}(\rho, \lambda)\left\lceil i\right.$ name for a Sacks tree contained in $q_{\alpha}(i)$ with stem containing $\dot{s}\left(q_{\alpha}, i, \alpha, \zeta\right)^{\frown} m, m<2$. Let $\dot{C}\left(\tilde{r}^{m}(i, \alpha, \zeta)\right)$ be a $\operatorname{Sacks}(\rho, \lambda)\lceil i$ name for the splitting levels of $\tilde{r}^{m}(i, \alpha, \zeta)$. Let $\dot{C}(i, \alpha)$ be a $\operatorname{Sacks}(\rho, \lambda)\lceil i$ name for the intersection of the $\dot{C}\left(\tilde{r}^{m}(i, \alpha, \zeta)\right), m<2, \zeta \in 2^{\alpha}$. Let $r^{m}(i, \alpha, \zeta)$ be a $\operatorname{Sacks}(\rho, \lambda)\left\lceil i\right.$ name for a restriction of $\tilde{r}^{m}(i, \alpha, \zeta)$ which splits exactly on levels in $\dot{C}(i, \alpha)$. Let $r_{\alpha+1}(i)=\bigcup\left\{r^{m}(i, \alpha, \zeta): m<2, \zeta \in 2^{\alpha}\right\}$. Then $r_{\alpha+1}(i)$ is a $\operatorname{Sacks}(\rho, \lambda)\lceil i$ name for an element of $\operatorname{Sacks}(\rho)$. Let

$$
q_{\alpha+1}=\left(q _ { \alpha } \lceil i _ { 0 } ) \frown r _ { \alpha + 1 } ( i _ { 0 } ) \frown \left(q _ { \alpha } \lceil ( i _ { 0 } , i _ { 1 } ) ) \frown r _ { \alpha + 1 } ( i _ { 1 } ) \frown \left(q_{\alpha}\left\lceil\left(i_{1}, i_{2}\right)\right) \frown \ldots\right.\right.\right.
$$

where $\left\langle i_{k}: k<\right.$ o.t. $\left.\left(X_{\alpha}\right)\right\rangle$ is the increasing enumeration of $X_{\alpha}$. Then $q_{\alpha+1} \in$ $\operatorname{Sacks}(\rho, \lambda)$, in fact $q_{\alpha+1} \in \tilde{D}$, and $q_{\alpha+1} \leq_{\alpha, X_{\alpha}} q_{\alpha}$. For limit ordinals $\alpha<\rho$, let $q_{\alpha}=\bigcap\left\{q_{\beta}: \beta<\alpha\right\}$. Let $q=\bigcap\left\{q_{\alpha}: \alpha<\rho\right\}$. Take the $X_{\alpha+1}$ 's so that $\bigcup\left\{X_{\alpha}: \alpha<\rho\right\}=\operatorname{supp}(q)$. This can be done as follows: For each $\beta<\rho$, once $q_{\beta}$ is chosen, enumerate $\operatorname{supp}\left(q_{\beta}\right)$ as $\left\{l_{\gamma}^{\beta}: \gamma<\rho\right\}$. At stage $\alpha+1<\rho$, make sure that $X_{\alpha+1}$ contains $\left\{l_{\gamma}^{\beta}: \beta \leq \alpha, \gamma \leq \alpha\right\}$. Then $q \in \operatorname{Sacks}(\rho, \lambda)$, and for each $\alpha<\rho, q \leq_{\alpha, X_{\alpha}} q_{\alpha}$.

This method allows one to obtain fusion sequences of length $\rho$ with certain desired properties, for instance, such that the $\tilde{r}^{m}(i, \alpha, \zeta)$ are in some dense set.

FACT 2.7. Assume $\rho<\lambda, \rho$ is strongly inaccessible, and $\lambda$ is weakly compact (or just strongly inaccessible). Then $\operatorname{Sacks}(\rho, \lambda)$ is $\rho$-closed, satisfies generalized $\rho$-fusion, preserves all cardinals $\leq \rho^{+}$, collapses $\lambda$ to $\rho^{++}$, is $\lambda$-c.c. so preserves all cardinals $\geq \lambda$, and blows up $2^{\rho}$ to $\rho^{++}$.

Fact 2.7 is proved by a straightforward modification of arguments in [2] for the analogous facts about $\operatorname{Sacks}\left(\omega, \omega_{2}\right)$.

We now give a very useful lemma about $\operatorname{Sacks}(\rho, \lambda)$. This lemma holds for any regular cardinal $\lambda>\rho$.

Lemma 2.8. Suppose $2^{\rho}=\rho^{+}, \rho$ is strongly inaccessible, and $\lambda>\rho$ is a regular cardinal. Let $\mathbb{S}$ denote $\operatorname{Sacks}(\rho, \lambda)$. Given $p \in \mathbb{S}, S \subseteq \lambda$ such that $|S|<\rho$, and $\alpha<\rho$, there exist $\gamma<\rho$, a club $C \subseteq \rho$, and $q \leq_{\alpha, S} p$ such that for each $i \in S, q\left\lceil i \Vdash\left(C \subseteq C(q(i))\right.\right.$ and $\left.\operatorname{ht}\left(\operatorname{Split}_{\alpha}(q(i))\right) \leq \gamma\right)$, where by $\mathrm{ht}\left(\operatorname{Split}_{\alpha}(q(i))\right)$ we mean the supremum of the ordinals decided to be the height of $\operatorname{Split}_{\alpha}(q(i))$ by some $r \leq q$. (Equivalently, for each $i \in S$, $q \uparrow i \Vdash C \subseteq C(q(i))$ and the $\alpha$ th element of $C(q(i))$ is $\leq \gamma$.) 
Proof. Let $p, S, \alpha$ be given as in the hypotheses. Without loss of generality, assume $p \in \tilde{D}$.

Claim. For each $r \in \mathbb{S}$, there is an $r^{\prime} \leq r$ in $\mathbb{S}$ and a club $C \subseteq \rho$ such that for each $i \in S, r^{\prime} \uparrow i \Vdash C \subseteq C(r(i))$.

Proof. Let $\left\langle i_{k}: k<\delta\right\rangle$ be the strictly increasing enumeration of $S$, where $\delta=$ o.t. $(S)$. There is an $\left.r_{0}^{*} \in \mathbb{S}\right\rceil i_{0}$ such that $r_{0}^{*} \leq r\left\lceil i_{0}\right.$ and there is a club $C_{0} \subseteq \rho$ such that $r_{0}^{*} \Vdash C_{0} \subseteq C\left(r\left(i_{0}\right)\right)$. (This is because $\mathbb{S}\left\lceil i_{0}\right.$ has $\rho$-fusion.) Let $r_{0}=r_{0}^{*} \frown\left(r \uparrow\left[i_{0}, \lambda\right)\right)$. Again, there exists an $r_{1}^{*} \in \mathbb{S}\left\lceil i_{1}\right.$ such that $r_{1}^{*} \leq r_{0}\left\lceil i_{1}\right.$ and there is a club $C_{1} \subseteq \rho$ such that $r_{1}^{*} \Vdash C_{1} \subseteq C\left(r\left(i_{1}\right)\right)$ (since $r\left(i_{1}\right)$ is exactly $\left.r_{0}\left(i_{1}\right)\right)$. Let $r_{1}=r_{1}^{*} \frown\left(r_{0}\left\lceil\left\lceil i_{1}, \lambda\right)\right)\right.$, which is the same as $r_{1}^{*} \frown\left(r \uparrow\left[i_{1}, \lambda\right)\right)$. Then $r_{1} \leq r_{0}$. In general, for $k<\delta$, take $r_{k+1}^{*} \in \mathbb{S}\left\lceil i_{k+1}\right.$ such that $r_{k+1}^{*} \leq r_{k}\left\lceil i_{k+1}\right.$ and a club $C_{k+1} \subseteq \rho$ such that $r_{k+1}^{*} \Vdash C_{k+1} \subseteq$ $C\left(r\left(i_{k+1}\right)\right)$. Let $r_{k+1}=r_{k+1}^{*} \frown\left(r\left\lceil\left[i_{k+1}, \lambda\right)\right)\right.$. For limit $k<\delta$, let $r_{k}^{\prime}=\bigcap_{l<k} r_{l}$. Then there are an $r_{k}^{*} \in \mathbb{S}\left\lceil k\right.$ such that $r_{k}^{*} \leq r_{k}^{\prime}\left\lceil i_{k}\right.$ and a club $C_{k} \subseteq \rho$ such that $r_{k}^{*} \Vdash C_{k} \subseteq C\left(r\left(i_{k}\right)\right)$. Let $r_{k}=r_{k}^{*} \frown\left(r\left\lceil\left[i_{k}, \lambda\right)\right)\right.$. In the end, let $r^{\prime}=\bigcap_{k<\delta} r_{k}$. Since $\delta<\rho$, we have $r^{\prime} \in \mathbb{S}$. Moreover, for each $\left.i \in S, r^{\prime}\right\rceil i \Vdash C_{i} \subseteq C(r(i))$. Let $C=\bigcap_{i \in S} C_{i}$. Then for each $i \in S, r^{\prime} \mid i \Vdash C \subseteq C(r(i))$.

To prove the lemma, we will use $\left(2^{\alpha+1}\right)^{S}$ many different combinations of choices on the indices in $S$ to preserve splitting up to level $i$ on indices in $S$. List the elements of $\left(2^{\alpha+1}\right)^{S}$ as $\left\langle\vec{\eta}_{\beta}: \beta<\left|\left(2^{\alpha+1}\right)^{S}\right|\right\rangle$ so that each element repeats cofinally often.

Given a condition $p$, let $\dot{s}(p, i, \alpha, \varepsilon)$ be a canonical total $\mathbb{S} \nmid i$ name for the $\varepsilon$ th element of the collection of immediate successors of elements in $\operatorname{Split}_{\alpha}(p(i))$, for each $\varepsilon \in 2^{\alpha+1}$. Let $p(i)\lceil\dot{s}(p, i, \alpha, \varepsilon)$ denote a total $\mathbb{S}\lceil i$ name for all nodes in $p(i)$ which are compatible with $\dot{s}(p, i, \alpha, \varepsilon)$.

Now we create a fusion sequence. Let $q_{0}=p$. Given $q_{\beta} \in \tilde{D}$ for some $\beta<\left|\left(2^{\alpha+1}\right)^{S}\right|$, we find a $q_{\beta+1} \leq_{\alpha, S} q_{\beta}$ in the following manner. First, let $r_{\beta}^{\prime}$ denote the condition defined as follows. For each $i \in S$, let $r_{\beta}^{\prime}(i)$ equal $q_{\beta}(i)\left\lceil\dot{s}\left(q_{\beta}, i, \alpha, \vec{\eta}_{\beta}(i)\right)\right.$, and for each $i \in \lambda \backslash S$, let $r_{\beta}^{\prime}(i)$ equal $q_{\beta}(i)$. Then $r^{\prime} \in \tilde{D}$, since $r \in \tilde{D}$. Now, using the Claim, take an $r_{\beta} \leq r_{\beta}^{\prime}$ such that there is a club $C_{\beta} \subseteq \rho$ such that for each $i \in S, r_{\beta}\left\lceil i \Vdash C_{\beta} \subseteq C\left(q_{\beta}(i)\right)\right.$. Then put back the other branches as in the Fusion Method Using Canonical Names to obtain a $q_{\beta+1} \leq_{\alpha, S} q_{\beta}$ with the property that whenever one restricts $q_{\beta+1}(i)$ through $\dot{s}\left(q_{\beta}, i, \alpha, \vec{\eta}_{\beta}(i)\right)$ for all $i \in S$, the resulting condition is $r_{\beta}$. When $\beta$ is a limit ordinal, then let $q_{\beta}$ be the intersection of all $q_{\beta^{\prime}}, \beta^{\prime}<\beta$.

Let $q=\bigcap\left\{q_{\beta}: \beta<\left|\left(2^{\alpha+1}\right)^{S}\right|\right\}$ and $C=\bigcap\left\{C_{\beta}: \beta<\left|\left(2^{\alpha+1}\right)^{S}\right|\right\}$. Then $q \leq_{\alpha, S} p$. Suppose $\beta<\left|\left(2^{\alpha+1}\right)^{S}\right|$ and $q^{\prime} \leq q$ is any condition such that for each $i \in S, q^{\prime}(i) \leq q(i)\left\lceil\dot{s}\left(q, i, \alpha, \vec{\eta}_{\beta}(i)\right)\right.$. Then $q^{\prime} \leq r_{\beta}$, so $q^{\prime}\left\lceil i \Vdash C \subseteq C\left(q_{\beta}(i)\right)\right.$ for each $i \in S$. Since each $\vec{\eta} \in\left(2^{\alpha+1}\right)^{S}$ appears cofinally in the listing, for a cofinal subsequence of $\beta$ 's, the above holds. Therefore, for each $i \in S$, 
$q^{\prime}\left\lceil i \Vdash C \subseteq C(q(i))\right.$. Since this is true for a dense set of $q^{\prime}$ below $q$, it follows that for each $i \in S, q\lceil i \Vdash C \subseteq C(q(i))$. Letting $\gamma$ be the $\alpha$ th element of $C$, we obtain the Lemma.

Lemma 2.9. Suppose $2^{\rho}=\rho^{+}, \rho$ is strongly inaccessible, and $\lambda>\rho$ is a regular cardinal. ( $\lambda$ need not be weakly compact, nor even inaccessible here.) Let $\mathbb{S}$ denote $\operatorname{Sacks}(\rho, \lambda)$. For each $p \in \mathbb{S}$, each $S \in[\lambda]^{<\rho}$, and each $i<\rho$, there exist $q \leq_{i, S} p$ and $\alpha<\rho$ such that for each $k \in S, q\lceil k \Vdash(\forall t \in$ $\left.\operatorname{Split}_{i}(q(k)), \operatorname{ht}(t)=\alpha\right)$.

Proof. We know from Lemma 2.8 that there is a $q \leq_{i, S} p$ and a ground model club $C \subseteq \rho$ such that for each $k \in S, q\lceil k \Vdash C \subseteq C(q(k))$. Let $\alpha$ be the $i$ th member of $C$. List $S=\left\langle k_{0}, k_{1}, \ldots\right\rangle$ in increasing order. Since $q\left\lceil k_{0} \Vdash C \subseteq\right.$ $C\left(q\left(k_{0}\right)\right)$, there is an $\mathbb{S}\left\lceil k_{0}\right.$ name $r\left(k_{0}\right)$ such that $q \uparrow k_{0} \Vdash r\left(k_{0}\right) \subseteq q\left(k_{0}\right)$ and $C\left(r\left(k_{0}\right)\right) \cap(\alpha+1)=C \cap(\alpha+1)$. Let $q_{0}=\left(q\left\lceil k_{0}\right) \frown r\left(k_{0}\right) \frown\left(q \uparrow\left(k_{0}, \lambda\right)\right) . q_{0} \in \mathbb{S}\right.$, and since $q_{0}\left\lceil k_{1} \leq q\left\lceil k_{1}\right.\right.$, also $q_{0}\left\lceil k_{1} \Vdash C \subseteq C\left(q\left(k_{1}\right)\right)\right.$. Since $q\left(k_{1}\right)=q_{0}\left(k_{1}\right)$, we see that $q_{0} \backslash k_{1} \Vdash C \subseteq C\left(q_{0}\left(k_{1}\right)\right)$. Take $r\left(k_{1}\right)$ to be an $\mathbb{S}\left\lceil k_{1}\right.$ name such that $q_{0}\left\lceil k_{1} \Vdash r\left(k_{1}\right) \subseteq q_{0}\left(k_{1}\right)\right.$ and $C\left(r\left(k_{1}\right)\right) \cap(\alpha+1)=C \cap(\alpha+1)$. Let $q_{1}=$ $\left(q_{0}\left\lceil k_{1}\right) \frown r\left(k_{1}\right) \frown\left(q \uparrow\left(k_{1}, \lambda\right)\right)\right.$. In this manner, we get a decreasing sequence $q_{k}$, $k \in S$. Let $q=\bigcap_{k \in S} q_{k}$. Then for each $k \in S, q\lceil k \Vdash C(q(k)) \cap(\alpha+1)=$ $C \cap(\alpha+1)$. Thus, for each $k \in S, q \uparrow k \Vdash \forall t \in \operatorname{Split}_{i}(q(k))$, ht $(t)=\alpha$.

3. Sacks $(\rho, \lambda)$ produces the tree property at $\rho^{++}$. The point of this section is Theorem 3.2. This result is stated without proof in [15]. As a self-contained proof is not available in the literature, and as our methods differ somewhat from those of Baumgartner and Laver in [2] and Kanamori in [15], we provide a proof here.

We begin with a general lemma.

Lemma 3.1. Let $\rho$ be a cardinal. Suppose $\mathbb{P}$ is a $\rho$-closed partial ordering. Suppose $\dot{B}$ is a $\mathbb{P}$-name for a sequence of length $\rho^{++}$not in $V$ such that for each $\xi<\rho^{++}, \mathbb{P} \Vdash \dot{B}\left\lceil\xi \in V\right.$. Let $p \in \mathbb{P}$. Then there is a $\gamma<\rho^{++}$such that $|\{x \in V: \exists q \leq p(q \Vdash \dot{B} \mid \gamma=x)\}| \geq \rho$.

Proof. Since $\dot{B}$ is a name for a sequence not in $V, p$ does not decide all of $\dot{B}$. So there are $\gamma_{0}<\delta$ and $p_{0}, q_{0} \leq p$ which force different values of $\dot{B}\left\lceil\gamma_{0}\right.$. Let $\gamma_{1}>\gamma_{0}$ be least such that $q_{0}$ does not decide $\dot{B}\left\lceil\gamma_{1}\right.$. Then there are $p_{1}, q_{1} \leq q_{0}$ which force different values of $\dot{B}\left\lceil\gamma_{1}\right.$. Given $q_{k}, \gamma_{k}$, let $\gamma_{k+1}>\gamma_{k}$ be least such that $q_{k}$ does not decide $\dot{B}\left\lceil\gamma_{k+1}\right.$. Then there are $p_{k+1}, q_{k+1} \leq q_{k}$ which force different values of $\dot{B}\left\lceil\gamma_{k+1}\right.$. At limit $k<\rho$, let $q_{k}=\inf \left\{q_{l}: l<k\right\}$ and $\gamma_{k}=\sup \left\{\gamma_{l}: l<k\right\} ; q_{k}$ exists, since $\mathbb{P}$ is $\rho$-closed. Continuing in this manner, we obtain $p_{k+1}, q_{k}, \gamma_{k}, k<\rho$, such that

(1) $\left\langle\gamma_{k}: k<\rho\right\rangle$ is a strictly increasing sequence of ordinals below $\rho^{++}$.

(2) For all $k<\rho, p_{k+1}$ and $q_{k+1}$ decide different values for $\dot{B}\left\lceil\gamma_{k+1}\right.$. 
(3) For $k<l, p_{l+1} \leq q_{k+1}$, which implies that $p_{k+1}$ and $p_{l+1}$ decide different values for $\dot{B}\left\lceil\gamma_{k+1}\right.$.

Let $\gamma=\sup _{k<\rho} \gamma_{k}$. Then $\gamma<\rho^{++}$and all $p_{k+1}, k<\rho$, disagree about the value of $\dot{B}\left\lceil\gamma\right.$; that is, for all $k<l<\rho$, for any $r \leq p_{k+1}$ and any $q \leq p_{l+1}$ such that $r$ and $q$ each decide $\dot{B}\lceil\gamma, r$ and $q$ must force different values for $\dot{B}\left\lceil\gamma_{k}\right.$, and hence for $\dot{B}\lceil\gamma$.

THEOREM 3.2. Let $\rho$ be a strongly inaccessible cardinal and $\lambda$ be a weakly compact cardinal above $\rho$. Let $\mathbb{S}$ denote $\operatorname{Sacks}(\rho, \lambda)$. Then in $V^{\mathbb{S}}, \lambda=\rho^{++}=$ $2^{\rho}$ and $\rho^{++}$has the tree property.

Proof. Let $G$ be $\mathbb{S}$-generic. By Fact 2.7, $\lambda=\rho^{++}=2^{\rho}$ in $V[G]$. Suppose, towards a contradiction, that $T$ is a $\rho^{++}$-Aronszajn tree in $V[G]$. For the sake of clarity, we remark that each node of $T$ is an increasing sequence of ordinals less than $\rho^{++}$. Let $\dot{T}$ be an $\mathbb{S}$ name for $T$. We may assume that $\dot{T}$ has size $\lambda$. Since $\lambda$ is weakly compact in $V$, there is an elementary embedding $j: M \rightarrow N$ with critical point $\lambda$ such that $\dot{T} \in M, M$ and $N$ are transitive $\mathrm{ZF}^{-}$-models, and $M \supseteq V_{\lambda}$. Then $j(\dot{T}) \in N$, so since $\lambda \in N$, we have $\dot{T}=j(\dot{T}) \cap \lambda \in N$. Therefore $T \in N[G]$. As $T$ has no cofinal branch in $V[G]$, it has none in $N[G]$; that is, $T$ is an Aronszajn tree in $N[G]$.

The forcing $j(\mathbb{S})$ is $N$ 's version of the $j(\lambda)$-length iteration of $\operatorname{Sacks}(\rho)$ with supports of size at most $\rho$. (That is, $N$ 's version of $\operatorname{Sacks}(\rho, j(\lambda))$.) The forcing $j(\mathbb{S})$ factors as $\mathbb{S} * \dot{\mathbb{R}}$, where $\dot{\mathbb{R}}$ is a term for a partial ordering which is isomorphic to $\operatorname{Sacks}(\rho, j(\lambda))$ defined over $N^{\mathbb{S}}$. To avoid ambiguity, we consider the iteration $\dot{\mathbb{R}}$ as indexed on the interval $[\lambda, j(\lambda))$. Let $\mathbb{R}$ denote $\dot{\mathbb{R}}^{G}$ in $N[G]$. Choose $H$ to be $\mathbb{R}$-generic over $N[G]$ (in some outer universe; $H$ cannot exist in $V[G]$, where $\lambda$ is a cardinal, as $H$ introduces a collapse of $\lambda$ to $\rho^{+}$). Then the embedding $j: M \rightarrow N$ lifts (in some outer universe) to $j^{*}: M[G] \rightarrow N[G][H]$. As $T$ is an initial segment of the tree $j^{*}(T)$, it follows that $T$ has a cofinal branch in $N[G][H]$. However, this contradicts the following Lemma 3.3 .

Lemma 3.3. The forcing $\mathbb{R}$ for adding $H$ over $N[G]$ adds no cofinal branch through $T$.

Proof. Suppose not. Let $\dot{B}$ denote a new branch through $T$ in $N[G][H]$. We work in $N[G]$.

The following lemma will give us tools to construct, using $\dot{B}$, a subtree of $T$ which has $2^{\rho}$ many branches of some length less than $\rho^{++}$, leading to a contradiction.

Lemma 3.4. Work in $N[G]$. Let $p \in \mathbb{R}, X \subseteq[\rho, j(\lambda))$ with $|X|<\rho$, and $\alpha<\rho$. Let $\delta=$ o.t. $(X)$ and $d=\left|2^{(\alpha+1) \delta}\right|$. We can find $q^{0}, q^{1} \leq_{\alpha, X} p$, an increasing sequence of ordinals $\left\langle\gamma_{k}: k<d\right\rangle$, a sequence $\left\langle Z_{k+1}: k<d\right\rangle$ such 
that each $Z_{k+1} \subseteq T$ is in $N[G]$ and has size less than $\rho$, and $\left\langle y_{k+1}: k<d\right\rangle$ elements of $T$ such that the following hold:

$$
\begin{aligned}
& \left\{y_{k+1}: k<d\right\} \cap \bigcup\left\{Z_{k+1}: k<d\right\}=\emptyset, \\
& q^{0} \Vdash\left(\forall k<d, \dot{B}\left\lceil\gamma_{k} \in Z_{k+1}\right),\right. \\
& q^{1} \Vdash\left(\exists k<d \text { such that } \dot{B}\left\lceil\gamma_{k}=y_{k+1}\right) .\right.
\end{aligned}
$$

Proof. Recall that since $T$ is a tree in $N[G]$ of height $\rho^{++}$, for any $\xi<\rho^{++}, \dot{B}\lceil\xi$ is a name for a sequence of length $\xi$ in $N[G]$ of ordinals less than $\rho^{++}$.

Let $p \in \mathbb{R}$. We start by setting some notation for the proof of this lemma. Given $i \in[\rho, j(\lambda))$, let $\mathbb{R}_{i}$ denote $\mathbb{R} \uparrow[\rho, i)$. Recall that we work in $\tilde{D}$. Let $\left\langle i_{\varepsilon}: \varepsilon<\delta\right\rangle$ be the strictly increasing enumeration of $X$. Let $i_{\delta}=$ $\sup \left\{i_{\varepsilon}: \varepsilon<\delta\right\}$. We have $\delta<\rho$ since $|X|<\rho$. Next, $p \in \tilde{D}$ implies that for each $\varepsilon<\delta$, there are $\mathbb{R}_{i_{\varepsilon}}$ names $\dot{s}_{\varepsilon, \zeta}\left(\zeta \in 2^{\alpha+1}\right)$ such that $\mathbb{R}_{i_{\varepsilon}} \Vdash\left(\dot{s}_{\varepsilon, \zeta}\right.$ is the $\zeta$ th node of $\left.\operatorname{Split}_{\alpha+1}\left(p\left(i_{\varepsilon}\right)\right)\right)$, where the nodes of $\operatorname{Split}_{\alpha+1}\left(p\left(i_{\varepsilon}\right)\right)$ are ordered canonically lexicographically. Let $\dot{t}_{\varepsilon, \zeta}^{0}=\dot{s}_{\varepsilon, \zeta} \frown 0$ and $\dot{t}_{\varepsilon, \zeta}^{1}=\dot{s}_{\varepsilon, \zeta} \frown 1$. Then each $\dot{t}_{\varepsilon, \zeta}^{m}(m<2)$ is an $\mathbb{R}_{i_{\varepsilon}}$ name for the $m$ th extension of the $\zeta$ th splitting node of $\operatorname{Split}_{\alpha+1}\left(p\left(i_{\varepsilon}\right)\right)$. Let $\left\langle u_{k}: k<d\right\rangle$ enumerate $\left(2^{\alpha+1}\right)^{\delta}$ (the $\delta$-length sequences whose entries are elements of $\left.2^{\alpha+1}\right)$ so that each $u_{k}=\left\langle u_{k}(\varepsilon): \varepsilon<\delta\right\rangle$, where each $u_{k}(\varepsilon) \in 2^{\alpha+1}$.

The idea of the proof is as follows. We use $\left|2^{(\alpha+1) \delta}\right|=d$ many Stages $\mathbf{k}$ $(k<d)$ each with $d$ many Substages k.l $(l<d)$ in order to construct the desired $q^{0}, q^{1}, \gamma_{k}, Z_{k+1}, y_{k+1}(k<d) . q^{1}$ and $q^{0}$ are not treated symmetrically. We construct a $d \times d$ length fusion sequence to construct $q^{0}$, whereas only a $d$ length fusion sequence to construct $q^{1}$.

We use the Stages $\mathbf{k}$ to construct sequences $\left\langle p_{k}^{0}: k<d\right\rangle$ and $\left\langle p_{k}^{1}: k<d\right\rangle$ such that the following hold.

(1) For all $k<k^{\prime}<d, p_{k^{\prime}}^{0} \leq_{\alpha, X} p_{k}^{0}$ and $p_{k^{\prime}}^{1} \leq_{\alpha, X} p_{k}^{1}$. Hence, $p^{0}=\inf \left\{p_{k}^{0}\right.$ : $k<d\} \leq_{\alpha, X} p$ and $p^{1}=\inf \left\{p_{k}^{1}: k<d\right\} \leq_{\alpha, X} p$.

(2) For each $k<d$, whenever $p_{k}^{1}$ is thinned on all indices $i_{\varepsilon} \in X$ through $u_{k}(\varepsilon)$, which represents a particular member of $\operatorname{Split}_{\alpha}\left(p_{k}^{1}(i)\right)$, the resulting condition decides a value for $\dot{B}\left\lceil\gamma_{k}\right.$. That is, whenever $r \leq$ $p_{k}^{1}$ satisfies $r\left\lceil i_{\varepsilon} \Vdash r\left(i_{\varepsilon}\right) \subseteq p_{k}^{1}\left(i_{\varepsilon}\right)\left\lceil\dot{t}_{\varepsilon, u_{k}(\varepsilon)}^{1}\right.\right.$, then $r \Vdash \dot{B}\left\lceil\gamma_{k}=y_{k+1}\right.$. In particular, the condition " $p_{k}^{1}$ thinned through $\dot{t}_{u_{k}}^{1}$ ", which we will denote $q_{k+1}^{1}$, satisfies the previous sentence. (See Fact 3.5.)

(3) $p_{k}^{0}$ and $Z_{k+1}$ satisfy the following: $y_{k+1} \notin Z_{k+1}$ and $p_{k}^{0} \Vdash \dot{B}\left\lceil\gamma_{k} \in\right.$ $Z_{k+1}$. To obtain such a $p_{k}^{0}$ and $Z_{k+1}$, we use Substages k.l $(l<d)$ to create a sequence $\left\langle p_{k, l}^{0}: l<d\right\rangle$ such that for each $l<l^{\prime}<d$, $p_{k, l^{\prime}}^{0} \leq_{\alpha, X} p_{k, l}^{0}$; moreover, for each $l<d$, whenever we restrict $p_{k, l}^{0}$ through $\dot{t}_{u_{l}}^{0}$, this condition forces $\dot{B}\left\lceil\gamma_{k}\right.$ to be in $Z_{k+1}$. 
The following fact will be used throughout the construction. For $m<2$, we say that $q \leq_{\alpha, X}^{m} p$ if $q \leq_{\alpha, X} p$ and for each $\varepsilon<\delta, q\left\lceil i_{\varepsilon} \Vdash\left(\forall \zeta \in 2^{\alpha+1}, \dot{t}_{\varepsilon, \zeta}^{m} \in\right.\right.$ $\left.q\left(i_{\varepsilon}\right)\right)$. That is, $q\left(i_{\varepsilon}\right)$ and $p\left(i_{\varepsilon}\right)$ both have the same $m$ th extensions of all $(\alpha+1)$ st splitting nodes of $p\left(i_{\varepsilon}\right)$.

FACT 3.5. Suppose $q \in \tilde{D}, m<2, q \leq_{\alpha, X}^{m} p$, and $k<d$. We can construct a condition $r$ called $q$ thinned through $\dot{t}_{u_{k}}^{m}$, denoted by $(q)^{t_{u_{k}}^{m}}$, where $r$ is the name obtained in the following manner: $r\left\lceil i_{0}=q\left\lceil i_{0}\right.\right.$; for each $\varepsilon<\delta$, $r\left(i_{\varepsilon}\right)=q\left(i_{\varepsilon}\right)\left\lceil\dot{t}_{\varepsilon, u_{k}(\varepsilon)}^{m}\right.$ and $r\left\lceil\left(i_{\varepsilon}, i_{\varepsilon+1}\right)=q \uparrow\left(i_{\varepsilon}, i_{\varepsilon+1}\right)\right.$; and $r\left\lceil\left[i_{\delta}, \lambda\right)=q \uparrow\left[i_{\delta}, \lambda\right)\right.$. Then $r \in \tilde{D}$, and for each $\varepsilon<\delta, r\left\lceil i_{\varepsilon} \Vdash r\left(i_{\varepsilon}\right)=q\left(i_{\varepsilon}\right)\left\lceil i_{u_{k}\left(i_{\varepsilon}\right)}^{m}\right.\right.$ up to the $(\alpha+1)$ st splitting level of $q\left(i_{\varepsilon}\right)$; i.e. $\operatorname{stem}\left(r\left(i_{\varepsilon}\right)\right)=$ the $u_{k}\left(i_{\varepsilon}\right)$ th element of $\operatorname{Split}_{\alpha+1}\left(q\left(i_{\varepsilon}\right)\right)^{\frown} m$.

Now we begin the construction of $q^{0}$ and $q^{1}$.

Stage 0. Let $p_{0}^{0}=p_{0}^{1}=p$.

Stage $\mathbf{k}+\mathbf{1}(k<d)$. Given $p_{k}^{0}, p_{k}^{1} \in \tilde{D}$ such that $p_{k}^{0} \leq_{\alpha, X}^{0} p$ and $p_{k}^{1} \leq_{\alpha, X}^{1} p$, let $q_{k+1}^{1}$ denote $p_{k}^{1}$ restricted through $\dot{t}_{u_{k}}^{1}$; i.e. $\left(p_{k}^{1}\right)^{\dot{t}_{u_{k}}^{1}}$. By Fact 3.5. $q_{k+1}^{1}$ is in $\tilde{D}$. Note that for all $i \in[\rho, j(\lambda)), \mathbb{R}_{i} \Vdash q_{k+1}^{1}(i) \leq p(i)$. Let $\gamma_{k}$ be such that $p_{k+1}^{1}$ can decide $\dot{B}\left\lceil\gamma_{k}\right.$ in $\rho$ many different ways. Such a $\gamma_{k}$ exists by Lemma 3.1 .

The following fact will be used to make sure we can continue after each substage.

FACT 3.6. Suppose $m<2, p^{\prime} \in \tilde{D}$ is such that $p^{\prime} \leq_{\alpha, X}^{m} p$ and $r \in \tilde{D}$ is such that $r \leq\left(p^{\prime}\right)^{\dot{t}_{u_{k}}^{m}}$. Then there is a $p^{\prime \prime} \in \tilde{D}$ such that $p^{\prime \prime} \leq_{\alpha, X}^{m} p^{\prime}$ and $\left(p^{\prime \prime}\right)^{\dot{t}_{u_{k}}^{m}} \sim r$. We say that $p^{\prime \prime}$ is $p^{\prime}$ refined through $\dot{t}_{u_{k}}^{m}$ to $r$.

Substage $\mathbf{k}+1$ 1.0. Let $q_{k+1,0}^{0}$ denote $\left(p_{k}^{0}\right)^{\dot{t}_{u_{0}}^{0}}$, that is, $p_{k}^{0}$ restricted through $\dot{t}_{u_{0}}^{0}$. Let $r_{k+1,0}^{0} \leq q_{k+1,0}^{0}$ be such that $r_{k+1,0}^{0} \in \tilde{D}$ and $r_{k+1,0}^{0}$ decides $\dot{B}\left\lceil\gamma_{k}\right.$, say as $z_{k+1,0}$. Let $p_{k+1,0}^{0}$ be $p_{k}^{0}$ refined through $u_{0}$ to $r_{k+1,0}^{0}$, as provided by Fact 3.6. Then $p_{k+1,0}^{0} \leq_{\alpha, X}^{0} p_{k}^{0} \leq_{\alpha, X}^{0} p$. Note that if $r \leq p_{k+1,0}^{0}$ and for each $\varepsilon<\delta, r\left\lceil i_{\varepsilon} \Vdash\left(r\left(i_{\varepsilon}\right)\right.\right.$ is a tree with stem $\left.\supseteq \dot{t}_{\varepsilon, u_{0}(\varepsilon)}^{0}\right)$, then $r \leq r_{k+1,0}^{0}$; hence, $r \Vdash \dot{B}\left\lceil\gamma_{k}=z_{k+1,0}\right.$.

Substage $\mathbf{k}+1 . l+1(l<d)$. Given $p_{k+1, l}^{0} \in \tilde{D}$ such that $p_{k+1, l}^{0} \leq_{\alpha, X}^{0}$ $p_{k+1, n}^{0}$ for all $n<l$, let $q_{k+1, l+1}^{0}=\left(p_{k+1, l}^{0}\right)^{\dot{t}_{u_{l}}^{0}}$, the restriction of $p_{k+1, l}^{0}$ through $\dot{t}_{u_{l}}^{0}$. Let $r_{k+1, l+1}^{0} \leq q_{k+1, l+1}^{0}$ be an element of $\tilde{D}$ which decides a value, call it $z_{k+1, l+1}$, for $\dot{B}\left\lceil\gamma_{k}\right.$. Let $p_{k+1, l+1}^{0}$ be $p_{k+1, l}^{0}$ refined through $\dot{t}_{u_{l}}^{0}$ to $r_{k+1, l+1}^{0}$, by Fact 3.6. Then $p_{k+1, l+1}^{0} \in \tilde{D}$ and $p_{k+1, l+1}^{0} \leq_{\alpha, X}^{0} p_{k+1, l}^{0}$. Note: If $r \leq p_{k+1, l+1}^{0}$ and for each $\varepsilon<\delta, r\left\lceil i_{\varepsilon} \Vdash\left(\operatorname{stem}\left(r\left(i_{\varepsilon}\right)\right) \supseteq \dot{t}_{\varepsilon, u_{l}(\varepsilon)}^{0}\right)\right.$, then $r \leq r_{k+1, l+1}^{0}$. Hence, $r \Vdash \dot{B}\left\lceil\gamma_{k}=z_{k+1, l+1}\right.$. 
Substage $\mathbf{k}+\mathbf{1} . l$ (for $l<d$ a limit). Let $p_{k+1, l}^{0}=\inf \left\{p_{k+1, n}^{0}: n<l\right\}$. Then $p_{k+1, l}^{0} \leq_{\alpha, X}^{0} p_{k}^{0}$, since $\mathbb{R}$ is $\rho$-closed and for each $\varepsilon<\delta$ and each $n<l$, $\mathbb{R}_{i_{\varepsilon}} \Vdash p_{k+1, n}^{0}\left(i_{\varepsilon}\right) \leq_{\alpha, X}^{0} p\left(i_{\varepsilon}\right)$.

At the end of all the Substages $\mathbf{k}+1 . l(l<d)$, let $p_{k+1}^{0}=\inf \left\{p_{k+1, l}^{0}\right.$ : $l<d\}$ and $Z_{k+1}=\left\{z_{k+1, l}: l<d\right\}$. Then $p_{k+1}^{0} \leq_{\alpha, X}^{0} p_{k}^{0}$. Note that $p_{k+1}^{0} \Vdash$ $\dot{B}\left\lceil\gamma_{k} \in Z_{k+1}\right.$.

To finish Stage $\mathbf{k}+\mathbf{1}$, choose an $r_{k+1}^{1} \leq q_{k+1}^{1}$ such that $r_{k+1}^{1}$ decides $\dot{B}\left\lceil\gamma_{k}\right.$ to be $y_{k+1}$ for some $y_{k+1} \notin Z_{k+1}$. Let $p_{k+1}^{1}$ be $p_{k}^{1}$ refined through $\dot{t}_{u_{k}}^{1}$ to $r_{k+1}^{1}$. Note: Any $q \leq p_{k+1}^{1}$ which has, for each $\varepsilon<\delta, q\left\lceil i_{\varepsilon} \Vdash\left(\operatorname{stem}\left(q\left(i_{\varepsilon}\right)\right) \supseteq\right.\right.$ $\left.\dot{t}_{\varepsilon, u_{k}(\varepsilon)}^{1}\right)$ will decide that $\dot{B}\left\lceil\gamma_{k}=y_{k+1}\right.$.

Stage $\mathbf{k}(k<d$ a limit). At limit $k<d$, we have decreasing sequences $\left\langle p_{l}^{0}: l<k\right\rangle$ and $\left\langle p_{l}^{1}: l<k\right\rangle$ such that for each $l<l^{\prime}<k$ and each $m<2$, $p_{l^{\prime}}^{m} \leq_{\alpha, X}^{m} p_{l}^{m}$. Thus, letting $p_{k}^{m}=\inf \left\{p_{l}^{m}: l<k\right\}$, we have $p_{k}^{m} \leq_{\alpha, X}^{m} p_{l}^{m}$ for all $l<k$.

At the end of all the Stages, for each $m<2$, let $q^{m}=\inf \left\{p_{k}^{m}: k<d\right\}$. Since for each $k<d, p_{k+1}^{m} \leq_{\alpha, X}^{m} p_{k}^{m}$, we have $p^{m} \leq_{\alpha, X}^{m} p$.

By construction, $q^{0} \Vdash\left(\forall k<d, \dot{B}\left\lceil\gamma_{k} \in Z_{k+1}\right)\right.$, and $q^{1} \Vdash(\exists k<d$ such that $\dot{B}\left\lceil\gamma_{k}=y_{k+1}\right)$.

This ends the proof of Lemma 3.4 .

Claim. The width of $T$ at some level below $\rho^{++}$is at least $2^{\rho}$.

Proof. We induct on $\operatorname{lh}(s)$ in order to define conditions extending $p$. Let $I_{\emptyset}=\left\{i_{\emptyset . \zeta}: \zeta<\rho\right\}$ be an enumeration of $\operatorname{supp}(p)$. Let $X_{\emptyset}=\left\{i_{\emptyset, 0}\right\}$. By Lemma 3.4. there are $q_{\langle 0\rangle}, q_{\langle 1\rangle} \leq_{0, X_{\emptyset}} p, \gamma_{\emptyset}<\rho^{++}$, and $Y_{\emptyset} \subseteq T$ in $N[G]$ such that $q_{\langle 1\rangle} \Vdash\left(\exists y \in Y_{\emptyset}\right.$ such that $\dot{B}\left\lceil\gamma_{\emptyset} \supseteq y\right)$, and $q_{\langle 0\rangle} \Vdash\left(\forall y \in Y_{\emptyset}, \dot{B}\left\lceil\gamma_{\emptyset} \nsupseteq y\right)\right.$.

Suppose now we are given $s \in 2^{<\rho}, q_{s} \in \tilde{D}$, and $\gamma_{s}$. Let $I_{s}=\left\{i_{s, \zeta}: \zeta<\rho\right\}$ be an enumeration of $\operatorname{supp}\left(q_{s}\right)$. Let $X_{s}=\left\{i_{s \uparrow l, \zeta}: l, \zeta \leq \operatorname{lh}(s)\right\}$. Then $\left|X_{s}\right|<\rho$. By Lemma 3.4, there are $q_{s \frown 0}, q_{s \frown 1} \leq_{\operatorname{lh}(s), X_{s}} q_{s}, \gamma_{s}>\gamma_{s \mid l}$ for all $l<\operatorname{lh}(s)$, $Y_{s} \subseteq T$ in $N[G]$, and a sentence $\varphi_{s} \equiv$ " $\exists y \in Y_{s}$ such that $\dot{B}\left\lceil\gamma_{s}\right.$ extends $y$ " such that $q_{s \frown 0} \Vdash \neg \varphi_{s}$ and $q_{s \frown 1} \Vdash \varphi_{s}$. For $s \in 2^{<\rho}$ with $\operatorname{lh}(s)$ a limit ordinal, let $q_{s}=\inf \left\{q_{s \mid l}: l<\operatorname{lh}(s)\right\}$.

Let $\gamma^{*}=\sup \left\{\gamma_{s}: s \in 2^{<\rho}\right\}$. Then $\gamma^{*}<\rho^{++}$. Let $b \in 2^{\rho}, b \in N[G]$. The sequence $\left\langle q_{b\lceil l}: l<\rho\right\rangle$ is in $N[G]$, so by generalized $\rho$-fusion, $q_{b}=\inf \left\{q_{b\lceil l}\right.$ : $l<\rho\} \in N[G]$. Then $q_{b}$ forces $\dot{B}\left\lceil\gamma^{*}\right.$ to extend or not extend (depending on the value of $b(l))$ an element of $Y_{b \mid l}$. Since $\gamma^{*}<\rho^{++}$, for each $b \in 2^{\rho}$ we can choose an extension $r_{b} \leq q_{b}$ such that $r_{b}$ decides a value for $\dot{B}\left\lceil\gamma^{*}\right.$. Then for all $b, c \in N[G]$ such that $b \neq c$ and $b, c \in 2^{\rho}, r_{b}$ and $r_{c}$ decide different values for $\dot{B}\left\lceil\gamma^{*}\right.$. Therefore, the tree $T$ has size at least $2^{\rho}$ at level $\gamma^{*}$, and $2^{\rho}=\rho^{++}$in $N[G]$. This concludes the proof of the Claim. 
The Claim contradicts that $T$ is a $\rho^{++}$-tree in $N[G]$. Thus, $\mathbb{R}$ cannot add a new branch through $T$ over $N[G]$. This concludes the proof of Lemma 3.3 .

By Lemma 3.3. $T$ has no cofinal branch in $N[G][H]$. But this contradicts that the embedding $j$ could be lifted to $j^{*}: M[G] \rightarrow N[G][H]$. Therefore, there is no $\rho^{++}$-Aronszajn tree in $V[G]$.

4. The tree property at the double successor of a measurable cardinal from a weakly compact hypermeasurable cardinal. In this section, we show that starting with a weakly compact hypermeasurable cardinal $\kappa$, there is a forcing extension in which $\kappa$ remains measurable and the tree property holds at $\kappa^{++}$. Recall Definition 1.1 of a weakly compact hypermeasurable cardinal.

TheOREM 4.1. Suppose GCH holds and $\kappa$ is a weakly compact hypermeasurable cardinal in $V$. Then there is a forcing extension $V[G]$ of $V$ in which $\kappa$ is still measurable and the tree property holds at $\kappa^{++}$.

Proof. Assume GCH in $V$ and let $\kappa$ be a weakly compact hypermeasurable cardinal in $V$. We define, in $V, \rho_{k}$ and $\lambda_{k}$ for $k \leq \kappa$ and a forcing notion $\mathbb{P}$ as follows. Let $\rho_{0}$ be the first inaccessible cardinal. Let $\lambda_{0}$ be the least weakly compact cardinal above $\rho_{0}$. For $k<\kappa$, given $\lambda_{k}$, let $\rho_{k+1}$ be the least inaccessible cardinal above $\lambda_{k}$. Let $\lambda_{k+1}$ be the least weakly compact cardinal above $\rho_{k+1}$. For limit ordinals $k \leq \kappa$, let $\rho_{k}$ be the least inaccessible cardinal greater than or equal to $\sup _{l<k} \lambda_{l}$. Let $\lambda_{k}$ be the least weakly compact cardinal above $\rho_{k}$. Note that $\rho_{\kappa}=\kappa$, and $\lambda_{\kappa}$ is the least weakly compact cardinal above $\kappa$.

Let $\mathbb{P}_{0}$ be the trivial forcing. For $i \leq \kappa$, if $i=\rho_{k}$ for some $k \leq \kappa$, let $\dot{\mathbb{Q}}_{i}$ be a $\mathbb{P}_{i}$-name for the sum (see Chapter 5 of $[6]$ ) of $\operatorname{Sacks}\left(\rho_{k}, \lambda\right)$ where $\lambda$ ranges over all inaccessible cardinals greater than $\rho$ and less than or equal to $\lambda_{k}$. In other words, let $\dot{\mathbb{Q}}_{i}$ be a $\mathbb{P}_{i}$ name for $\bigoplus A=\{\langle\mathbb{Q}, p\rangle: \mathbb{Q} \in A$ and $p \in \mathbb{Q}\} \cup\{\mathbf{1}\}$, ordered with $\mathbf{1}$ above everything else and $\left\langle\mathbb{Q}^{\prime}, p^{\prime}\right\rangle \leq\langle\mathbb{Q}, p\rangle$ when $\mathbb{Q}^{\prime}=\mathbb{Q}$ and $p^{\prime} \leq p$, where $A=\left\{\operatorname{Sacks}(\rho, \lambda): \rho<\lambda \leq \lambda_{k}\right.$ and $\lambda$ is inaccessible $\}$; otherwise, let $\dot{\mathbb{Q}}_{i}$ be a $\mathbb{P}_{i}$ name for the trivial forcing. Let $\mathbb{P}_{i+1}=\mathbb{P}_{i} * \dot{\mathbb{Q}}_{i}$. Let $\mathbb{P}=\mathbb{P}_{\kappa+1}$ be the iteration $\left\langle\left\langle\mathbb{P}_{i}, \dot{\mathbb{Q}}_{i}\right\rangle: i \leq \kappa\right\rangle$ with reverse Easton support. In our notation, $\mathbb{P}=\mathbb{P}_{\kappa+1}=\mathbb{P}_{\kappa} * \dot{\mathbb{Q}}_{\kappa}$.

We choose to force below a condition which forces $\mathbb{P}_{\kappa} * \dot{\mathbb{Q}}_{\kappa}$ to be forcing equivalent to $\mathbb{P}_{\kappa} * \operatorname{Sacks}\left(\kappa, \lambda_{\kappa}\right)$. Let $G$ be $\mathbb{P}_{\kappa}$-generic over $V$. Take $g$ to be generic for $\dot{\mathbb{Q}}_{\kappa}^{G}$ over $V[G]$. Then $G * g$ is $\mathbb{P}$-generic over $V$. By Theorem 3.2 , $\operatorname{TP}\left(\kappa^{++}\right)$holds in $V[G][g]$. In fact, $\operatorname{TP}\left(\rho_{k}^{++}\right)$holds in $V[G][g]$ for all $k<\kappa$. (Note that $\left\{\rho_{k}: k<\kappa\right\}$ is a set of measure 1 in $\kappa$ for any normal measure on $\kappa$.)

The rest of the proof consists of proving that $\kappa$ remains measurable in $V[G][g]$. To this end, let $j: V \rightarrow M$ be an elementary embedding witnessing 
the weakly compact hypermeasurability of $\kappa$. We can assume that $j$ is given by a hyperextender ultrapower so that $M=\{j(f)(a): f \in V, f: H(\kappa) \rightarrow V$, and $\left.a \in H\left(\lambda_{\kappa}\right)\right\}$, and $V$ and $M$ have the same $H\left(\lambda_{\kappa}\right)$. For consider $T=$ $\left\{j(f)(a): f \in V, f: H(\kappa) \rightarrow V\right.$, and $\left.a \in H\left(\lambda_{\kappa}\right)\right\}$. Then $T \prec M$ contains $H\left(\lambda_{\kappa}\right)$ as a subset. Let $\pi$ be the transitive collapse of $T \cong$ onto $\bar{M}$. Then $\pi \circ j$ is a hyperextender ultrapower embedding witnessing the weakly compact hypermeasurablility of $\kappa$.

We need to find a suitable generic for $j(\mathbb{P})$ over $M$ in order to lift $j$ to $V[G][g]$. Since $M$ and $V$ have the same $H\left(\lambda_{\kappa}\right), j(\mathbb{P})_{\kappa}$ (the first $\kappa$ stages of $j(\mathbb{P})$ in $M)$ is equal to $\mathbb{P}_{\kappa}$ and so $G$ is also generic for $j(\mathbb{P})_{\kappa}$ over $M$. Let $j(\mathbb{P})(\kappa)$ denote the $(\kappa+1)$ st stage of $j(\mathbb{P})$, and let $\left(\lambda_{\kappa}\right)^{M}$ denote the least weakly compact cardinal in $M$ above $\kappa$. Note that in $M[G], j(\mathbb{P})(\kappa)$ is the sum of $\left\{\operatorname{Sacks}(\kappa, \lambda): \kappa<\lambda \leq\left(\lambda_{\kappa}\right)^{M}\right.$ and $\lambda$ is inaccessible $\}$. Again, since $M$ and $V$ have the same $H\left(\lambda_{\kappa}\right)$ and therefore $\lambda_{\kappa} \leq\left(\lambda_{\kappa}\right)^{M}, g$ is also generic for $j(\mathbb{P})(\kappa)$ over $M[G]$.

REMARK 4.2. If $\kappa$ is the least weakly compact hypermeasurable in $V$ then in fact $\left(\lambda_{\kappa}\right)^{M}$ is greater than $\lambda_{\kappa}$. To see this, suppose that $j: V \rightarrow M$ witnesses that $\kappa$ is weakly compact hypermeasurable in $V$, and let $\lambda$ be the least weakly compact cardinal in $V$ greater than $\kappa$. Then $o(\kappa)$ is at least $\lambda$ in $M$, as $V$ and $M$ have the same $H(\lambda)$. If $\lambda$ were weakly compact in $M$, then $o(\kappa)$ would be greater than $\lambda$ in $M$. (See the reflection argument given in the last paragraph of the proof of Theorem 5.3.) Then $\kappa$ would be weakly compact hypermeasurable in $M$, contradicting our assumption that $\kappa$ was the least weakly compact hypermeasurable in $V$. It is for this reason that we used not $\operatorname{Sacks}\left(\rho_{k}, \lambda_{k}\right)$ but rather a sum of forcings $\operatorname{Sacks}\left(\rho_{k}, \lambda\right)$ at stage $\rho_{k}$ of the reverse Easton iteration $\mathbb{P}$.

Let $j(\mathbb{P})\lceil(\kappa, j(\kappa))$ denote the forcing $j(\mathbb{P})$ restricted to indices above $\kappa+1$ and below $j(\kappa)$. That is, $j(\mathbb{P})\lceil(\kappa, j(\kappa))$ is the remainder in $M$ of the forcing $j\left(\mathbb{P}_{\kappa}\right)$ above $\mathbb{P}_{\kappa+1}$. To obtain a generic $H$ for $j(\mathbb{P})\lceil(\kappa, j(\kappa))$ over $M[G][g]$, we argue as follows, similarly to [10].

Each open dense subset of $j(\mathbb{P}) \uparrow(\kappa, j(\kappa))$ in $M[G][g]$ is of the form $j(f)(a)^{G * g}$, where $a \in H\left(\lambda_{\kappa}\right), f: H(\kappa) \rightarrow V$ and $f \in V$. Since $j(\mathbb{P})\lceil j(\kappa) \subseteq$ $H(j(\kappa))^{M}$, every open dense subset of $j(\mathbb{P})\lceil(\kappa, j(\kappa))$ in $M[G][g]$ is an element of $\left(H\left(j(\kappa)^{+}\right)\right)^{M[G][g]}$. Thus, we can assume that $f: H(\kappa) \rightarrow H\left(\kappa^{+}\right)$. $j(\mathbb{P})\left\lceil(\kappa, j(\kappa))\right.$ begins with $\mathbb{Q}_{\kappa}$, which is the sum of $\operatorname{Sacks}\left(\rho_{\kappa+1}, \lambda\right)$ over all inaccessible $\lambda \in\left(\rho_{\kappa+1}, \lambda_{\kappa+1}\right]$ in $M[G][g]$, where $\rho_{\kappa+1}$ denotes the least inaccessible cardinal in $M$ above $\left(\lambda_{\kappa}\right)^{M}$. Hence, $j(\mathbb{P})\left\lceil(\kappa, j(\kappa))\right.$ is $\rho_{\kappa+1}$-closed in $M[G][g]$. Since $\rho_{\kappa+1}>\left(\lambda_{\kappa}\right)^{M} \geq \lambda_{\kappa}$ and there are only $\lambda_{\kappa}$ many open dense subsets of $j(\mathbb{P})\left\lceil(\kappa, j(\kappa))\right.$ of the form $j(f)(a)^{G * g}, a \in H\left(\lambda_{\kappa}\right)$, there is a single condition in $j(\mathbb{P})\lceil(\kappa, j(\kappa))$ in $M[G][g]$ which meets all open dense sets of the form $j(f)(a)^{G * g}, a \in H\left(\lambda_{\kappa}\right)$. 
Since GCH holds in $V$, there are only $\kappa^{+}$many such functions $f: H(\kappa) \rightarrow$ $H\left(\kappa^{+}\right)$. List them as $\left\langle f_{i}: i<\kappa^{+}\right\rangle$. For each $i+1<\kappa^{+}$, let $p_{i+1}$ be an element of $j(\mathbb{P}) \uparrow(\kappa, j(\kappa))$ satisfying $p_{i+1} \leq p_{i}$ and $p_{i+1}$ meets every open dense set $j\left(f_{i}\right)(a)^{G * g}, a \in H\left(\lambda_{\kappa}\right)$. For each limit ordinal $i<\kappa^{+}$, by the analogous form of Lemma 3 of [10] for iterated Sacks forcing, the sequence $\left\langle p_{i^{\prime}}: i^{\prime}<i\right\rangle$ is in $M[G][g]$. By $\rho_{\kappa+1}$-closure in $M[G][g]$, there is a $p_{i} \in j(\mathbb{P})\lceil(\kappa, j(\kappa))$ below every $p_{i^{\prime}}$ for all $i^{\prime}<i$. Let $H$ be the set of all elements of $j(\mathbb{P})\lceil(\kappa, j(\kappa))$ above some element of $\left\{p_{i}: i<\kappa^{+}\right\}$. Then $H$ is $j(\mathbb{P}) \uparrow(\kappa, j(\kappa))$-generic over $M[G][g]$.

Thus, we have a generic $G * g * H$ for $j\left(\mathbb{P}_{\kappa}\right)$ over $M$, and $j[G] \subseteq G * g * H$. Hence, the embedding $j$ lifts to an embedding $j^{*}: V[G] \rightarrow M[G][g][H]$. Let $M^{*}$ denote $M[G][g][H]$. Note that $j^{*}$ is definable in $V[G][g]$, not in $V[G]$.

For the final stage, we need to lift $j^{*}$ (in $V[G][g]$ ) to an elementary embedding $j^{* *}: V[G][g] \rightarrow M^{*}[h]$ for some $h$ generic for $j^{*}\left(\dot{\mathbb{Q}}_{\kappa}^{G}\right)$ over $M^{*}$. Since $g$ is generic for $\operatorname{Sacks}\left(\kappa, \lambda_{\kappa}\right)$ over $V[G]$, we need to find an $h$ which is generic for $\operatorname{Sacks}\left(j(\kappa), j\left(\lambda_{\kappa}\right)\right)$ over $M^{*}$.

To simplify notation, we make the following conventions and abuses of notation. Let $\lambda$ denote $\lambda_{\kappa}$. Let $\mathbb{Q}_{j(\kappa)}^{*}$ denote the last stage of the iteration $j(\mathbb{P})$ in $M^{*}$. Note that $\mathbb{Q}_{j(\kappa)}^{*}=j^{*}\left(\mathbb{Q}_{\kappa}\right)=j\left(\dot{\mathbb{Q}}_{\kappa}\right)^{G * g * H}$. We shall need to find an $h$ which is generic for $\mathbb{Q}_{j(\kappa)}^{*}$ over $M^{*}$ such that $j^{*}[g] \subseteq h$. Since $g$ is generic for $\operatorname{Sacks}(\kappa, \lambda)$ over $V[G]$, we shall need $h$ to be generic for $\operatorname{Sacks}(j(\kappa), j(\lambda))$ over $M^{*}$. We abuse notation and let $\mathbb{Q}_{\kappa}$ denote $\operatorname{Sacks}(\kappa, \lambda)$ in $V[G]$ and $\mathbb{Q}_{j(\kappa)}^{*}$ denote $\operatorname{Sacks}(j(\kappa), j(\lambda))$ in $M^{*}$ for the rest of the proof of the measurability of $\kappa$ in $V[G][g]$. For $i<\lambda$, $\mathbb{Q}_{\kappa}\lceil i$ denotes the $i$-length iteration of $\operatorname{Sacks}(\kappa)$ in $V[G]$ with supports of size $\leq \kappa$. For $i<j(\lambda)$, we let $\mathbb{Q}_{j(\kappa)}^{*}\lceil i$ denote the forcing obtained at the $i$ th stage of the iteration $\operatorname{Sacks}(j(\kappa), j(\lambda))$ in $M^{*}$; that is, the $i$-length iteration of $\operatorname{Sacks}(j(\kappa))$ in $M^{*}$ with $\leq j(\kappa)$-sized supports. Let $\tilde{D}_{\kappa}$ denote the collection of elements $p \in \mathbb{Q}_{\kappa}$ in $V[G]$ such that for all $i \leq \sup (\operatorname{supp}(p)), \mathbb{Q}_{\kappa}\lceil i \Vdash p(i) \in \operatorname{Sacks}(\kappa)$; that is, the trivial condition of $\mathbb{Q}_{\kappa} \uparrow i$ forces $p(i)$ to be an element of Sacks $(\kappa)$. Note that $\tilde{D}_{\kappa}$ is a dense subset of $\mathbb{Q}_{\kappa}$, and moreover, for each $p \in \mathbb{Q}_{\kappa}$, there is a $q \in \tilde{D}_{\kappa}$ such that $q \sim p$. (This follows from Fact 2.6. $\tilde{D}_{\kappa}$ is a dense subset of $\mathbb{Q}_{\kappa}$. In the statement of Fact 2.6, let $p$ be the trivial element of $\mathbb{P}$. Then Fact 2.6 implies that for any $q \in \mathbb{P}$, there is an $r \sim q$ such that $r \in \tilde{D}$. Thus, substituting $p$ for $q, q$ for $r$, and $\mathbb{Q}_{\kappa}$ for $\mathbb{P}$ above, we conclude that for each $p \in \mathbb{Q}_{\kappa}$, there is a $q \in \tilde{D}_{\kappa}$ such that $q \sim p$.)

The following lemmas will help us choose $h$ based on $j^{*}[g]$ and later they will be used to prove that indeed, $h$ is $\mathbb{Q}_{j(\kappa)}^{*}$ generic over $M^{*}$. Lemma 4.4 is relevant to the final paragraph of Section 5 . 
Lemma 4.3. $\{\kappa\}=\bigcap\{j(C): C \subseteq \kappa$ is club in $V\}=\bigcap\left\{j^{*}(C): C \subseteq \kappa\right.$ is club in $V[G]\}$.

See [10] or [7] for a proof.

Lemma 4.4. $M^{*}$ and $V[G][g]$ have the same $H\left(\kappa^{++}\right)$. Moreover, in $V[G][g]$, $M^{*}$ is closed under $\kappa$ sequences in $V[G][g]$.

Proof. Let $x$ be in $H\left(\kappa^{++}\right)$of $V[G][g]$. Recall that we are forcing below a condition, call it $\mathbf{p}$, which forces $\mathbb{P}_{\kappa} * \dot{\mathbb{Q}}_{\kappa}$ to be forcing equivalent to $\mathbb{P}_{\kappa} *$ $\operatorname{Sacks}(\kappa, \lambda)$. Hence, the collection of conditions in $\mathbb{P}_{\kappa} * \dot{\mathbb{Q}}_{\kappa}$ below $\mathbf{p}$ satisfies the $\lambda$-c.c., by Fact 2.7. Hence, there is a $z \in V$ such that $z \supseteq \operatorname{tr} \operatorname{cl}(x)$ and $|z|<\lambda$ in $V$. Now, $V$ and $M$ have the same $H(\lambda)$. Therefore, there is a $\theta<\lambda$ and an $f: z \rightarrow \theta$ which is a bijection in $M$. Let $y=f^{\prime \prime} x$. Then $x \in M[y]$ since $f \in M$ and $x=f^{-1}[y]$. We have $y \in V[G][g]$, since $f \in M \subseteq V$ and $x \in V[G][g]$. But $y \subseteq \theta$. Hence, $y \in M[G][g]$, since the $H(\lambda)$ of $V$ and $M$ are the same, and $G * g$ is both $\mathbb{P}$-generic over $V$ and $j(\mathbb{P})\lceil(\kappa+1)$-generic over $M$. Therefore, $x \in M[G][g]$. Hence, $V[G][g]$ and $M[G][g]$ have the same $H\left(\kappa^{++}\right)$. Thus, $V[G][g]$ and $M^{*}$ have the same $H\left(\kappa^{++}\right)$.

The second part follows as in the proof of Lemma 3 of [10].

Note: Once we construct the generic $h$ for $\mathbb{Q}_{j(\kappa)}$ over $M^{*}$, it will follow from the first part of the preceding lemma that $V[G][g]$ and $M[G][g][H][h]$ have the same $H\left(\kappa^{++}\right)$.

The next fact is a general lemma about elementary embeddings.

Lemma 4.5. Suppose $j: V \rightarrow M$ is an elementary embedding, $\operatorname{crit}(j)=\kappa$, $M=\{j(f)(a): f \in V, f: H(\kappa) \rightarrow V, a \in H(\zeta)\}$ where $\zeta \leq j(\kappa), X \in M$, and $|X|=j(\kappa)$ in $M$. Then $X \cap \operatorname{ran}(j)$ is also in $M$. Furthermore, for each $x$ in $H\left(\kappa^{++}\right)$in $M^{*}, x \cap \operatorname{ran}(j)$ has size at most $\kappa$ in $M^{*}$.

Proof. Case 1: $X \in \operatorname{ran}(j)$. Since $\operatorname{ran}(j) \prec M, M \models$ (There is a bijection between $X$ and $j(\kappa)$ ), and $j(\kappa) \in \operatorname{ran}(j)$, by elementarity $\operatorname{ran}(j) \models$ (There is a bijection between $X$ and $j(\kappa))$. Let $\pi \in \operatorname{ran}(j)$ be such that $\operatorname{ran}(j) \models$ $(\pi: X \rightarrow j(\kappa)$ is a bijection).

Claim 4.5.1. $\pi: \operatorname{ran}(j) \cap X \rightarrow \operatorname{ran}(j) \cap j(\kappa)$ is a bijection.

Proof. If $x \in X \cap \operatorname{ran}(j)$, then $x=j(y)$ for some $y \in V$. Moreover, $\pi \in$ $\operatorname{ran}(j)$ implies there is some $\sigma \in V$ such that $\pi=j(\sigma)$. So $\pi(x)=\pi(j(y))=$ $j(\sigma)(j(y))=j(\sigma(y))$. Therefore, $\pi(x) \in \operatorname{ran}(j) \cap j(\kappa)$. Furthermore, $\pi$ is 1-1. Let $z \in \operatorname{ran}(j) \cap j(\kappa)$. Then there is some $u \in V$ such that $z=j(u)$. Now, $z \in j(\kappa)$ implies $\pi^{-1}(z) \in X$. So $\pi^{-1}(z)=\pi^{-1}(j(u))=j\left(\sigma^{-1}\right)(j(u))=$ $j\left(\sigma^{-1}(u)\right) \in \operatorname{ran}(j) \cap X$. Therefore, $\pi \uparrow(\operatorname{ran}(j) \cap X)$ is a bijection between $\operatorname{ran}(j) \cap X$ and $\operatorname{ran}(j) \cap j(\kappa)$.

We have $\operatorname{ran}(j) \cap j(\kappa)=\kappa \in M$, and $\pi \in M$ implies $\pi^{-1} \in M$. Hence, $\pi^{-1} \uparrow(\operatorname{ran}(j) \cap j(\kappa)) \in M$. Therefore, $\pi^{-1}[\operatorname{ran}(j) \cap j(\kappa)]=\operatorname{ran}(j) \cap X \in M$. 
Case 2: $X \notin \operatorname{ran}(j)$. Still, $X=j(f)(b)$ for some $b \in H(\zeta)$ and some $f: H(\kappa) \rightarrow V$. We can assume that for all $\bar{a} \in \operatorname{dom}(f),|f(\bar{a})|=\kappa$. Then for all $a \in \operatorname{dom}(j(f)),|j(f)(a)|=j(\kappa)$. Let $Y=\bigcup\{j(f)(a): a \in H(j(\kappa))\}$. Then $|Y|=j(\kappa)$ and $Y \in \operatorname{ran}(j)$, since $H(j(\kappa))=j(H(\kappa)) \in \operatorname{ran}(j)$ and $j(f) \in \operatorname{ran}(j)$. Now, $b \in H(\zeta)$ where $\zeta \leq j(\kappa)$, so $X \subseteq Y$. Since $Y \in \operatorname{ran}(j)$ and $|Y|=j(\kappa)$, by Case $1, \operatorname{ran}(j) \cap Y \in M$. By our assumption, $X \in M$. Therefore, $\operatorname{ran}(j) \cap X=(\operatorname{ran}(j) \cap Y) \cap X \in M$.

Finally, we are ready to define $h$.

Definition of $h$. Let $\dot{\bar{x}}(i)$ denote the $\mathbb{Q}_{\kappa}\lceil i$ name $\bigcap\{\bar{p}(i): \bar{p} \in g\}$ in $V[G]$, for each $i<\lambda$. Let $\bar{x}(i)$ denote the element of $2^{\kappa}$ which $g \uparrow(i+1)$ forces $\dot{\bar{x}}(i)$ to be. For each $i \in j(\lambda) \cap \operatorname{ran}(j)$, letting $\bar{i}=j^{-1}(i)$, define $r_{i}$ to be the element of $\mathbb{Q}_{j(\kappa)}^{*}$ in $M^{*}$ such that for each $k \in j(\lambda) \backslash\{i\}, r_{i}(k)$ is a $\mathbb{Q}_{j(\kappa)}^{*} \backslash k$ name for the trivial condition $2^{<j(\kappa)}$, and $r_{i}(i)$ is a $\mathbb{Q}_{j(\kappa)}^{*} \backslash i$ name for the condition with stem $\bar{x}(\bar{i})^{\frown} 0$ and everywhere branching above; that is, $r_{i}(i)$ is a $\mathbb{Q}_{j(\kappa)}^{*}\lceil i$ name for the subtree of $2^{<j(\kappa)}$ consisting of all elements of $2^{<j(\kappa)}$ which are compatible with $\bar{x}(\bar{i}) \frown 0$.

Let $h$ be the filter generated in $V[G][g]$ by $j^{*}[g] \cup\left\{\bigcap_{i \in I} r_{i}: I \in M^{*}, I \subseteq\right.$ $j(\lambda) \cap \operatorname{ran}(j)$, and $|I| \leq j(\kappa)\}$. That is, first take all the possible intersections $j^{*}(p) \cap \bigcap_{i \in I} r_{i}$, where $p \in g$ and $I \subseteq j(\lambda) \cap \operatorname{ran}(j)$ for some $I \in M^{*}$ with $|I| \leq j(\kappa)$. Next take the upward closure of this set in $V[G][g]$. Then $h$ is well-defined in $V[G][g]$ since $j^{*}[g] \in V[G][g]$, and by Lemma 4.5, for each $I \in M^{*}$ such that $|I| \leq j(\kappa)$ in $M^{*}, I \cap \operatorname{ran}(j)$ is also in $M^{*}$ and has size at most $\kappa$ in $M^{*}$. This implies that any subset of $\operatorname{ran}(j)$ in $M^{*}$ of size at most $j(\kappa)$ actually has size at most $\kappa$ in $M^{*}$. Therefore, it suffices just to take all intersections of $\kappa$ many of the $r_{i}$ 's.

Main Claim. $h$ is generic for $\mathbb{Q}_{j(\kappa)}^{*}$ over $M^{*}$.

Proof. The idea of the proof is that, as in [10], the intersections of the elements in $j^{*}[g]$ should give us "tuning forks" (two cofinal branches in $2^{j(\kappa)}$ which branch exactly at height $\kappa)$ on indices in $j(\lambda) \cap \operatorname{ran}(j)$ and a cofinal branch in $2^{j(\kappa)}$ on indices in $j(\lambda) \backslash \operatorname{ran}(j)$. For a product $P$ of Sacks forcings, choosing the left branch at height $\kappa$ on indices in $j(\lambda) \cap \operatorname{ran}(j)$ yields a generic for $j(P)$ [10]. Unfortunately, this does not work for iterated Sacks forcing, since $j^{*}[g]$ only yields names for tuning forks and cofinal branches, and names do not help us show that $h$ meets every dense open subset of $\mathbb{Q}_{j(\kappa)}^{*}$. However, we will show that indeed, even in the case of iterated forcing, we can approximate the tuning forks and cofinal branches with concrete ground model objects. Toward this end we must do more work.

We give the following Definitions 4.6 and 4.8 and Lemma 4.7 in full generality for any iterated Sacks forcing. Definition 4.6 is the natural gen- 
eralization of the definition of " $(F, n)$-determined" in [2] for iterations of $\operatorname{Sacks}(\omega)$.

DeFinition 4.6. Let $\rho<\theta$ be cardinals with $\rho$ strongly inaccessible or $\omega$ in a ground model $W$ of ZFC in which $2^{\rho}=\rho^{+}$. Let $p \in \operatorname{Sacks}(\rho, \theta)$, $S \in[\theta]^{<\rho}$, and $\vec{x}=\langle x(l): l \in S\rangle$, where each $x(l) \in 2^{<\rho}$. Let $p(\vec{x})$ be obtained by induction on $l \in S$ by replacing $p(l)$ by all conditions in $p(l)$ which are compatible with $x(l)$. Precisely, for each $l \in S$, let $q(l)$ be the $\operatorname{Sacks}(\rho, l)$ name $\{\langle t, r\rangle \in p(l): t \subseteq x(l)$ or $t \supseteq x(l)\}$. Then let $p(\vec{x})$ be defined by $p(\vec{x})\left\lceil l_{0}=p\left\lceil l_{0}\right.\right.$, and for each $k<$ o.t. $(S), p(\vec{x})\left\lceil\left(l_{k}, l_{k+1}\right)=p\left\lceil\left(l_{k}, l_{k+1}\right)\right.\right.$ and $p(\vec{x})\left(l_{k}\right)=q\left(l_{k}\right)$, where $\left\langle l_{k}: k<\right.$ o.t. $\left.(S)\right\rangle$ is the increasing enumeration of $S$. Alternatively, one could define $r(l)=\{\langle t, r\rangle \in p(l): p(\langle x(k): k \in S \cap l\rangle) \Vdash$ $t \subseteq x(l)$ or $t \supseteq x(l)\}$ for each $l \in S$, and define $p(\vec{x})$ as above, substituting the $r(l)$ 's for $q(l)$ 's. (Note that $p(\vec{x})$ may or may not be a condition, but for our applications below for $(S, i)$-determined conditions, the above two definitions coincide and will produce a condition.)

Define $\vec{x}$ lies on $p$ by induction as follows: $\vec{x}$ lies on $p$ iff for each $l \in S$, $\vec{x} \uparrow(S \cap l)=\langle x(m): m \in S \cap l\rangle$ lies on $p$ and $p(\langle x(m): m \in S \cap l\rangle) \uparrow l \Vdash x(l) \in p(l)$.

It is easy to check that if $\vec{x}$ lies on $p$, then $p(\vec{x})$ is a condition in $\operatorname{Sacks}(\rho, \theta)$.

Suppose that $p \in \operatorname{Sacks}(\rho, \theta), S \in[\theta]^{<\rho}$, and $i<\rho$. We say that $p$ is $(S, i)$-determined iff for some $\alpha<\rho$,

(1) For each $k \in S, p\lceil k$ forces that nodes on the $i$ th splitting level of $p(k)$ have length $\alpha$.

(2) List $S$ as $k_{0}<k_{1}<\cdots$. Then $p\left(k_{0}\right) \cap 2^{\alpha+1}$ is an actual ground model tree of height $\alpha+1$. Choose any branch $x_{0}$ in $p\left(k_{0}\right) \cap 2^{\alpha+1}$. Then $p\left(\left\langle x_{0}\right\rangle\right)\left\lceil k_{1}\right.$ computes $p\left(k_{1}\right) \cap 2^{\alpha+1}$ as an actual ground model object. Choose any branch $x_{1}$ in this tree. Then the condition $p\left(\left\langle x_{0}, x_{1}\right\rangle\right)\left\lceil k_{2}\right.$ computes the tree $p\left(k_{2}\right) \cap 2^{\alpha+1}$ as an actual ground model object. In general, given $\left\langle x_{i}: i<l\right\rangle$ chosen in this manner, $p\left(\left\langle x_{i}: i<l\right\rangle\right)$ decides $p\left(k_{l}\right) \cap 2^{\alpha+1}$ as a ground model object. Let $x_{l}$ be a branch in this tree and continue in this manner for all indices $k$ in $S$.

It is clear that there are many $\vec{x}$ 's whose components have length $\alpha$ which lie on $p$ if $p$ is $(S, i)$-determined (with $i$ th splitting levels of length $\alpha$ ).

The purpose of $p$ being $(S, i)$-determined is that branches through the first $i$ splitting levels of the trees $p(k), k \in S$, are determined to be real ground model objects, and not names.

LEMMA 4.7. Let $\rho<\theta$ be cardinals with $\rho$ strongly inaccessible and $2^{\rho}=\rho^{+}$in a ground model $W$ of ZFC. Suppose $\bar{p} \in \operatorname{Sacks}(\rho, \theta), S \in[\theta]^{<\rho}$, and $i<\rho$. Then there is a $\bar{q} \leq_{i, S} \bar{p}$ such that $\bar{q}$ is $(S, i)$-determined.

Proof. Let $\bar{p} \in \operatorname{Sacks}(\rho, \theta)$ be given. By the proof of Lemma 2.9 there are $\bar{p}^{\prime} \leq_{i, S} \bar{p}$, an $\alpha<\rho$, and a club $C$ such that for each $k \in S$, 
$\bar{p}^{\prime} \nmid k \Vdash\left(C \cap(\alpha+1)=C\left(\bar{p}^{\prime}(k)\right) \cap(\alpha+1)\right.$, and $\left.\operatorname{ht}\left(\operatorname{Split}_{i}\left(\bar{p}^{\prime}(k)\right)\right)=\alpha\right)$. Enumerate $S=\left\langle k_{m}: m<\right.$ o.t. $\left.(S)\right\rangle$. Choose $\bar{p}^{\prime \prime}\left\lceil k_{0} \leq \bar{p}^{\prime} \uparrow k_{0}\right.$ such that $\bar{p}^{\prime \prime}\left\lceil k_{0}\right.$ decides $\bar{p}^{\prime}\left(k_{0}\right) \cap 2^{\alpha+1}$ as a tree in the ground model. List $\left(2^{i+1}\right)^{S}$ as $\left\langle\vec{\eta}_{l}: l<\left|\left(2^{i+1}\right)^{S}\right|\right\rangle$. Choose the $\vec{\eta}_{0}\left(k_{0}\right)$ th branch in the tree $\bar{p}^{\prime}\left(k_{0}\right) \cap 2^{\alpha+1}$ (by using the isomorphism between $\bar{p}^{\prime}\left(k_{0}\right)$ and $\left.2^{\alpha+1}\right)$. Let $\bar{p}^{\prime}\left(k_{0}\right)\left\lceil\vec{\eta}_{0}\left(k_{0}\right)\right.$ denote the subtree of nodes in $\bar{p}^{\prime}\left(k_{0}\right)$ which are comparable to the $\vec{\eta}_{0}\left(k_{0}\right)$ th element of $\bar{p}^{\prime}\left(k_{0}\right) \cap 2^{\alpha+1}$. Let $\bar{p}_{0}^{\prime \prime}\left\lceil k_{1}\right.$ denote $\left(\bar{p}^{\prime \prime}\left\lceil k_{0}\right) \frown\left(\bar{p}^{\prime}\left(k_{0}\right)\left\lceil\vec{\eta}_{0}\left(k_{0}\right)\right) \frown \bar{p}^{\prime} \uparrow\left(k_{0}, k_{1}\right)\right.\right.$. Take a $\bar{p}_{1}^{\prime \prime}\left\lceil k_{1} \leq \bar{p}_{0}^{\prime \prime} \uparrow k_{1}\right.$ such that $\bar{p}_{1}^{\prime \prime} \uparrow k_{1}$ decides $\bar{p}^{\prime}\left(k_{1}\right) \cap 2^{\alpha+1}$ as a tree in the ground model. Biject this ground model tree with $2^{\alpha+1}$ and choose the $\vec{\eta}_{0}\left(k_{1}\right)$ th branch. Let $\bar{p}_{1}^{\prime \prime}\left\lceil k_{2}\right.$ denote $\left(\bar{p}^{\prime \prime}\left\lceil k_{1}\right) \frown\left(\bar{p}^{\prime}\left(k_{1}\right)\left\lceil\vec{\eta}_{0}\left(k_{1}\right)\right) \frown\left(\bar{p}^{\prime} \uparrow\left(k_{1}, k_{2}\right)\right)\right.\right.$. Choose $\bar{p}_{2}^{\prime \prime}\left\lceil k_{2} \leq \bar{p}_{1}^{\prime \prime}\left\lceil k_{2}\right.\right.$ such that $\bar{p}_{2}^{\prime \prime}\left\lceil k_{2}\right.$ decides $\bar{p}^{\prime}\left(k_{2}\right) \cap 2^{\alpha+1}$ as a tree in the ground model. In this way ( $\operatorname{since} \operatorname{Sacks}(\rho, \theta)$ is $\rho$-closed), we construct a condition, call it $\bar{r}_{0} \leq \bar{p}^{\prime}$, such that for each $k \in S, \bar{r}_{0}\left\lceil k\right.$ decides $\bar{p}^{\prime}(k) \cap 2^{\alpha+1}$ as a tree in the ground model, and for each $k \in S, \bar{r}_{0}\left\lceil k \Vdash \operatorname{ht}\left(\operatorname{stem}\left(\bar{r}_{0}(k)\right)\right)>\alpha\right.$.

Now we wish to put back branches to get a $\bar{p}_{0} \leq_{i, S} \bar{p}^{\prime}$ in such a way that restricting $\bar{p}_{0}$ through $\vec{\eta}_{0}$ gives $\bar{r}_{0}$. Let $\bar{p}_{0}\left\lceil k_{0}=\bar{r}_{0}\left\lceil k_{0}\right.\right.$. For $\bar{p}_{0}\left(k_{0}\right)$, below $\bar{r}_{0}\left\lceil k_{0}\right.$, put back the trees above each of the nodes not equal to $\bar{r}_{0}\left(k_{0}\right) \cap$ $2^{\alpha+1}$ in $\bar{p}^{\prime}\left(k_{0}\right) \cap 2^{\alpha+1}$. Precisely, let $\bar{p}_{0}\left(k_{0}\right)=\bar{r}_{0}\left(k_{0}\right) \cup\left\{\left\langle\bar{p}^{\prime}\left(k_{0}\right)\left\lceil a, \bar{r}_{0}\left\lceil k_{0}\right\rangle\right.\right.\right.$ : $\bar{r}_{0}\left\lceil k_{0} \Vdash a \in \bar{p}^{\prime}\left(k_{0}\right) \cap 2^{\alpha+1}\right.$ and $a$ is not the $\vec{\eta}_{0}\left(k_{0}\right)$ th element of $\bar{p}^{\prime}\left(k_{0}\right) \cap$ $\left.2^{\alpha+1}\right\}$. Note that $\bar{r}_{0}\left\lceil k_{0} \Vdash \bar{p}_{0}\left(k_{0}\right) \in \operatorname{Sacks}(\rho)\right.$. Now $\bar{p}_{0}\left\lceil\left(k_{0}+1\right)\right.$ is a condition in $\operatorname{Sacks}\left(\rho, k_{0}+1\right)$. On $\left(k_{0}, k_{1}\right)$, let $\bar{p}_{0} \uparrow\left(k_{0}, k_{1}\right)$ be a $\operatorname{Sacks}\left(\rho, k_{0}+1\right)$ name such that $\bar{r}_{0} \uparrow\left(k_{0}+1\right)$ forces $\bar{p}_{0} \uparrow\left(k_{0}, k_{1}\right)=\bar{r}_{0} \uparrow\left(k_{0}, k_{1}\right)$, and each condition in $\operatorname{Sacks}\left(\rho, k_{0}+1\right)$ which is below $\bar{p}_{0} \uparrow\left(k_{0}+1\right)$ and is incompatible with $\bar{r}_{0} \uparrow\left(k_{0}+1\right)$ forces $\bar{p}_{0} \uparrow\left(k_{0}, k_{1}\right)=\bar{p}^{\prime} \uparrow\left(k_{0}, k_{1}\right)$. This gives us $\bar{p}_{0}\left\lceil k_{1}\right.$. Let $\bar{p}_{0}\left(k_{1}\right)$ be $\left\{\left\langle\bar{r}_{0}\left(k_{1}\right), \bar{r}_{0}\left\lceil k_{1}\right\rangle\right\} \cup\left\{\left\langle\bar{p}^{\prime}\left(k_{1}\right)\left\lceil a, \bar{r}_{0}\right\rceil k_{1}\right\rangle: \bar{r}_{0}\left\lceil k_{1} \Vdash a \in \bar{p}^{\prime}\left(k_{1}\right) \cap 2^{\alpha+1}\right.\right.\right.$ and $a$ is not the $\vec{\eta}_{0}\left(k_{1}\right)$ th element of $\left.\bar{p}^{\prime}\left(k_{1}\right) \cap 2^{\alpha+1}\right\} \cup\left\{\left\langle\bar{p}^{\prime}\left(k_{1}\right), b\right\rangle: b \in B\right\}$, where $B$ is a dense subset of elements below $\bar{p}_{0} \backslash k_{1}$ which are incompatible with $\bar{r}_{0} \backslash k_{1}$. Continuing in this way, we construct $\bar{p}_{0} \leq_{S, i} \bar{p}^{\prime}$ such that $\bar{p}_{0}$ forces nodes on the $i$ th splitting level of $\bar{p}_{0}(k)$ to have length $\alpha$, for each $k \in S$. Moreover, when we restrict $\bar{p}_{0}$ through the $\vec{\eta}_{0}(k)$ th branches for all $k \in S$, we get back $\bar{r}_{0}$; hence, for each $\gamma \in$ o.t. $(S), \bar{p}_{0}\left(\vec{\eta}_{0}\right)\left\lceil k_{\gamma}\right.$ computes $\bar{p}_{0}\left(k_{\gamma}\right) \cap 2^{\alpha+1}$ as a ground model object.

In this manner, we construct a decreasing sequence $\bar{p}_{l}, l<\left|\left(2^{i+1}\right)^{S}\right|$, such that for each such $l<l^{\prime}, \bar{p}^{\prime} \geq_{i, S} \bar{p}_{l} \geq_{i, S} \bar{p}_{l^{\prime}}$. More importantly, for each $l<\left|\left(2^{i+1}\right)^{S}\right|$, thinning $\bar{p}_{l}$ through $\vec{\eta}_{l}$ yields $\bar{r}_{l}$. Now, $\bar{r}_{l}$ has the properties that for each $k \in S, \bar{r}_{l}\left\lceil k \Vdash \bar{r}_{l}(k)\right.$ is a ground model object with all nodes on the $i$ th splitting level of $\bar{r}_{l}(k)$ having length $\alpha$; and for each $k \in S, \bar{r}_{l}\lceil k$ computes $\bar{p}_{l}(k)$ up to its $i$ th splitting level as a ground model object.

Let $\bar{q}=\bigcap_{l<\left|\left(2^{i+1}\right)^{S}\right|} \bar{p}_{l}$. Then $\bar{q} \leq_{i, S} \bar{p}$, and $\bar{q}$ is $(S, i)$-determined, with corresponding height $\alpha$.

Towards showing that $h$ is generic, let $D \in M^{*}$ be dense open in $\mathbb{Q}_{j(\kappa)}^{*}$. Write $D$ as $j^{*}(f)(\bar{a})$, where $f$ has domain $H(\kappa)^{V}, f \in$ 
$V[G]$, and $\bar{a} \in H(\lambda)^{V}$. (This is possible for the following reasons: Recall that $M=\{j(f)(a): f \in V, f: H(\kappa) \rightarrow V$, and $\left.a \in H(\lambda)^{V}\right\}$, and $j^{*}: V[G] \rightarrow M^{*}$ is defined by $j^{*}\left(\dot{x}^{G}\right)=$ $(j(\dot{x}))^{G * g * H}$, where $\dot{x}$ is a $\mathbb{P}$ name in $V$. Each element of $M^{*}$ is $\dot{y}^{G * g * H}$ for some $j(\mathbb{P})$ name $\dot{y}$ in $M$. For each such $\dot{y}$, there is a function in $V$, which we shall call $\dot{f}$, which takes values which are $\mathbb{P}$ names in $V$ such that $\dot{y}=j(\dot{f})(\check{a})$ for some $a \in H(\lambda)^{V}$. Then $\dot{y}^{G * g * H}=(j(\dot{f})(\check{a}))^{G * g * H}=j^{*}\left(\dot{f}^{G}\right)(a)$.) Without loss of generality, we can assume that $f(\bar{a})$ is a dense open subset of $\mathbb{Q}_{\kappa}$ in $V[G]$ for each $\bar{a} \in H(\lambda)^{V}$. The strategy is to show that any condition $\bar{p} \in$ $\mathbb{Q}_{\kappa}$ can be extended to "reduce" each of the $f(\bar{a}), \bar{a} \in H(\lambda)^{V}$, as in [10]. However, since we have an iteration, we need the following definition, which we give for a general iteration of Sacks forcings.

DeFinition 4.8. Let $\rho<\theta$ be cardinals with $\rho$ strongly inaccessible or $\omega$ and $2^{\rho}=\rho^{+}$in a ground model $W$ of ZFC. Let $p \in \operatorname{Sacks}(\rho, \theta), S \in[\theta]^{<\rho}$, and $i<\rho$. Suppose that $p$ is $(S, i)$-determined and $\alpha$ is the length of the nodes on the $i$ th splitting level of $p(k)$ for each $k \in S$. Then an $(S, i)$-thinning of $p$ is an extension of $p$ of the form $p(\vec{x})$, where $\vec{x}=\langle x(k): k \in S\rangle$ lies on $p$ and each $x(k)$ has length $\alpha+1$. We say that $p$ reduces the dense set $D$ iff for some $S$ and $i, p$ is $(S, i)$-determined and any $(S, i)$-thinning of $p$ meets $D$.

Let $\bar{p} \in \mathbb{Q}_{\kappa}$ and enumerate $H(\kappa)^{V}$ as $\left\langle\bar{a}_{k}: k<\kappa\right\rangle$. Using Lemmas 4.7 and 2.9, we can build a fusion sequence of conditions $\left\langle\bar{q}_{k}: k<\kappa\right\rangle$ below $\bar{p}$ such that for each $k<\kappa$, there is a set $\bar{S}_{k} \subseteq \lambda$ of size less than $\kappa$ such that

(1) $\bar{q}_{k+1} \leq_{k, \bar{S}_{k}} \bar{q}_{k}$.

(2) For each $k<\kappa, \bar{q}_{k+1}$ is $\left(\bar{S}_{k}, k\right)$-determined and any $\left(\bar{S}_{k}, k\right)$-thinning of $\bar{q}_{k+1}$ is a member of $f\left(\bar{a}_{k}\right)$.

(3) $\bigcup_{k<\kappa} \bar{S}_{k}=\operatorname{supp}(\bar{q})$, where $\bar{q}=\bigcap\left\{\bar{q}_{k}: k<\rho\right\}$.

To find such a $\bar{q}$, start by letting $\bar{q}_{0}=\bar{p}$. For $k<\kappa$, given $\bar{q}_{k}$ and $\bar{S}_{k} \subseteq \operatorname{supp}\left(\bar{q}_{k}\right)$ of size less than $\kappa$, use Lemma 2.9 to find a $\bar{q}_{k}^{\prime} \leq_{k, \bar{S}_{k}} \bar{q}_{k}$ and $\bar{\alpha}_{k}$ such that for each $m \in \bar{S}_{k}, \bar{q}_{k}^{\prime} \mid m \Vdash\left(\forall t \in \operatorname{Split}_{k}(\bar{q}(m))\right.$, ht $\left.(t)=\bar{\alpha}_{k}\right)$. Then use Lemma 4.7 to find a $\bar{q}_{k}^{\prime \prime} \leq_{k, \bar{S}_{k}} \bar{q}_{k}^{\prime}$ which is $\left(\bar{S}_{k}, k\right)$-determined. Enumerate $\left(2^{\bar{\alpha}_{k}+1}\right)^{S_{k}}$ as $\left\langle\vec{x}_{m}: 0<m<d\right\rangle$, where $d:=\left|\left(2^{\bar{\alpha}_{k}+1}\right)^{\bar{S}_{k}}\right|$. Let $\bar{q}_{k, 0}^{\prime \prime \prime}=\bar{q}_{k}^{\prime \prime}$. Given $\bar{q}_{k, m}^{\prime \prime \prime}$, take $\bar{r}_{m} \leq \bar{q}_{k, m}^{\prime \prime \prime}\left(\vec{x}_{m}\right)$ to be in $f\left(\bar{a}_{k}\right)$. Let $\bar{q}_{k, m+1}^{\prime \prime \prime}$ be the condition below $\bar{q}_{k, m}^{\prime \prime \prime}$ such that $\bar{q}_{k, m+1}^{\prime \prime \prime}\left(\vec{x}_{m}\right)=\bar{r}_{m}$ and for all $n \in \bar{S}_{k}, \bar{q}_{k, m+1}^{\prime \prime \prime}\lceil n$ forces that $\bar{q}_{k, m+1}^{\prime \prime \prime}(n) \cap 2^{\bar{\alpha}_{k}+1}=\bar{q}_{k, m}^{\prime \prime \prime}(n) \cap 2^{\bar{\alpha}_{k}+1}$ and that the tree $\bar{q}_{k, m+1}^{\prime \prime \prime}(n)$ minus the nodes lying above $\vec{x}_{m}(n)$ is equal to the tree $\bar{q}_{k, m}^{\prime \prime \prime}(n)$ minus the nodes lying above $\vec{x}_{m}(n)$. Then $\bar{q}_{k, m+1}^{\prime \prime \prime} \leq_{k, \bar{S}_{k}} \bar{q}_{k, m}^{\prime \prime \prime}$. For limit $m<d$, let $\bar{q}_{k, m}^{\prime \prime \prime}=\bigcap\left\{\bar{q}_{k, n}^{\prime \prime \prime}: n<m\right\}$. Then let $\bar{q}_{k+1}=\bigcap\left\{\bar{q}_{k, m}^{\prime \prime \prime}: m<d\right\}$. The point 
is that we can construct a $\bar{q}_{k+1} \leq_{k, \bar{S}_{k}} \bar{q}_{k}$ which is $\left(\bar{S}_{k}, k\right)$-determined with height $\bar{\alpha}_{k}$ such that for each $m<d, \bar{q}_{k+1}\left(\vec{x}_{m}\right)$ is an element of $f\left(\bar{a}_{k}\right)$; that is, $\bar{q}_{k+1}$ reduces $f\left(\bar{a}_{k}\right)$. Let $\bar{q}=\bigcap\left\{\bar{q}_{k}: k<\rho\right\}$. (As usual, make sure the sets $\bar{S}_{k}, k<\kappa$, were chosen so that $\bigcup\left\{\bar{S}_{k}: k<\kappa\right\}=\operatorname{supp}(\bar{q})$.)

Note that $\bar{q}$ reduces each dense set $f\left(\bar{a}_{k}\right)$ for each $k<\kappa$. As $g$ is generic, we can choose such a $\bar{q} \in g$. By elementarity, $q=j^{*}(\bar{q})$ reduces each dense set $j^{*}(f)(a)$ for each $a \in H(\lambda)^{V}$; so in particular, $q$ reduces $D$. Let $S$ and $i$ be such that $S$ is a subset of $j(\lambda)$ of size less than $j(\kappa), i<j(\kappa), q$ is $(S, i)$-determined, and any $(S, i)$-thinning of $q$ meets $D$. Let $\alpha$ be the length of the nodes on the $i$ th splitting level of $q(k)$ for $k \in S$.

The remaining problem becomes whether there is an $(S, i)$-thinning of $q$ which is in $h$. The following Lemma 4.9 will show that there is an $(S, i)$ thinning $r^{*}$ of $q$ which indeed is in $h$.

Lemma 4.9. Let $\bar{q} \in \mathbb{Q}_{\kappa}, S \subseteq j(\lambda)$ such that $|S|<j(\kappa)$, and $\alpha<j(\kappa)$ be given. Then there is an $\bar{r} \leq \bar{q}$ such that, letting $r$ denote $j^{*}(\bar{r})$,

(1) For each $k \in S, r \uparrow k$ determines $r(k) \cap 2^{\alpha+1}$ as a ground model object.

(2) For each $k \in S \cap \operatorname{ran}(j), r\lceil k \Vdash r(k)$ has no splitting between $\kappa$ and $\alpha+1$.

(3) For each $k \in S \backslash \operatorname{ran}(j), r\lceil k \Vdash \operatorname{ht}(\operatorname{stem}(r(k))) \geq \alpha+1$.

Hence, if $\bar{q} \in g$, then there is an $\bar{r} \leq \bar{q}$ such that $\bar{r} \in g$ and $j^{*}(\bar{r})$ satisfies (1)-(3). In our particular case, there is some $r^{*} \in h$ such that $r^{*} \leq q$ and for all $k \in S, r^{*}\left\lceil k\right.$ decides $r^{*}(k) \cap 2^{\alpha+1}$ to be an object in the ground model $M^{*}$.

Proof. Let $\bar{q} \in \mathbb{Q}_{\kappa}$. Let $\left\langle\bar{S}_{i}: i<\kappa\right\rangle$ be a continuous sequence of subsets of $\lambda$ of size less than $\kappa$ and $\left\langle\bar{\alpha}_{i}: i<\kappa\right\rangle$ be a continuous sequence of ordinals less than $\kappa$ such that the $(\kappa+1)$ st entry of $j^{*}\left(\left\langle\bar{S}_{i}: i<\kappa\right\rangle\right)$ contains $S$ and the $(\kappa+1)$ st entry of $j^{*}\left(\left\langle\bar{\alpha}_{i}: i<\kappa\right\rangle\right)$ is at least $\alpha$. Such sequences can be obtained as follows. Let $C$ be a club subset of $\kappa$ such that $j(C) \cap(\kappa, \alpha+1)=\emptyset$. Let $\left\langle\bar{\alpha}_{i}: i<\kappa\right\rangle$ be the increasing enumeration of $C . S=j^{*}(f)(a)$ for some $f: H(\kappa)^{V} \rightarrow[\lambda]^{<\kappa}$ in $V[G]$ and some $a \in H(\lambda)^{V}$. Define $\bar{S}_{i}=f^{\prime}(i)=$ $\bigcup\left\{f(\bar{b}): \bar{b} \in H\left(\bar{\alpha}_{i+1}\right)\right\}$. Then $j^{*}\left(f^{\prime}\right)(\kappa)=\bigcup\left\{j^{*}(f)(b): b \in H\left(\alpha_{\kappa+1}\right)\right\} \supseteq S$.

Let $\bar{q}_{0}=\bar{q}, \bar{T}_{0}=\emptyset$. Extend $\bar{q}_{0}$ to $\bar{q}_{1}$ and let $\bar{T}_{1}$ be such that $\left|\bar{T}_{1}\right|<\kappa$, $\bar{T}_{1}$ contains $\bar{S}_{1}$ and part of $\operatorname{supp}\left(\bar{q}_{0}\right), \bar{q}_{1}$ is $\left(\bar{T}_{1}, 1\right)$-determined, and for each $k \in \bar{T}_{1}, \bar{q}_{1}\left\lceil k\right.$ computes $\operatorname{stem}\left(\bar{q}_{1}(k)\right)$ as a ground model string of length $\geq$ $\bar{\alpha}_{1}+1$. (We can do this by first picking $\bar{q}_{1}^{\prime} \leq \bar{q}_{0}$ such that for each $k \in \bar{T}_{1}$, $\bar{q}_{1}^{\prime}\left\lceil k\right.$ computes $\operatorname{stem}\left(\bar{q}_{1}(k)\right)$ as a ground model string of length $\geq \bar{\alpha}_{1}+1$. Then by Lemma 4.7 take $\bar{q}_{1} \leq \bar{q}_{1}^{\prime}$ such that $\bar{q}_{1}$ is $\left(\bar{T}_{1}, 1\right)$-determined.)

Claim 4.9.1. Suppose $0<i<\kappa, \bar{q}_{i}, \bar{T}_{i}, \bar{T}_{i+1}$, and $\bar{\alpha}_{i+1}$ are such that $\bar{T}_{i+1} \supseteq \bar{T}_{i} \cup \bar{S}_{i+1} \cup\left(\right.$ part of $\left.\operatorname{supp}\left(\bar{q}_{i}\right)\right)$, and $C \subseteq \kappa$ is a club such that $j^{*}(C) \cap$ $(\kappa, \alpha+1)=\emptyset$. Then there are $\bar{\alpha}_{i+1}^{\prime} \geq \bar{\alpha}_{i+1}, \bar{q}_{i+1} \leq_{i, \bar{T}_{i}} \bar{q}_{i}$ such that

(i) $\bar{q}_{i+1}$ is $\left(\bar{T}_{i+1}, i+1\right)$-determined with corresponding height $\bar{\alpha}_{i+1}^{\prime}$. 
(ii) For each $k \in \bar{T}_{i+1} \backslash \bar{T}_{i}$, for each $\vec{x} \in\left(2^{\bar{\alpha}_{i+1}^{\prime}+1}\right)^{\bar{T}_{i+1}}$ which lies on $\bar{q}_{i+1}$, $\bar{q}_{i+1}(\vec{x})\left\lceil k \Vdash \operatorname{ht}\left(\operatorname{stem}\left(\bar{q}_{i+1}(\vec{x})(k)\right)\right) \geq \bar{\alpha}_{i+1}+1\right.$.

(iii) For each $k \in \bar{T}_{i+1}$, for each $\vec{x} \in\left(2^{\bar{\alpha}_{i+1}^{\prime}+1}\right)^{\bar{T}_{i+1}}$ which lies on $\bar{q}_{i+1}$, $\bar{q}_{i+1}(\vec{x})\left\lceil k \Vdash C\left(\bar{q}_{i+1}(\vec{x})(k)\right) \backslash\left(\bar{\alpha}_{i+1}+1\right) \subseteq C\right.$.

Proof. Let $C \subseteq \kappa$ be a club such that $j^{*}(C) \cap(\kappa, \alpha+1)=\emptyset$. By fusion, we can find a $\bar{q}_{i+1}^{\prime} \leq_{i, \bar{T}_{i}} \bar{q}_{i}$ such that for each $k \in \bar{T}_{i+1} \backslash \bar{T}_{i}, \bar{q}_{i+1}^{\prime}\lceil k \Vdash$ $\operatorname{ht}\left(\operatorname{stem}\left(\bar{q}_{i+1}^{\prime}\right)\right) \geq \bar{\alpha}_{i+1}+1$. Second, by fusion, find a $\bar{q}_{i+1}^{\prime \prime} \leq_{i, \bar{T}_{i}} \bar{q}_{i+1}^{\prime}$ such that for each $k \in \bar{T}_{i+1}, \bar{q}_{i+1}^{\prime \prime}\left\lceil k \Vdash C\left(\bar{q}_{i+1}^{\prime \prime}(k)\right) \backslash\left(\bar{\alpha}_{i+1}+1\right) \subseteq C\right.$. Third, by fusion again, find a $\bar{q}_{i+1}^{\prime \prime \prime} \leq_{i, \bar{T}_{i}} \bar{q}_{i+1}^{\prime \prime}$ such that for each $k \in \bar{T}_{i}, \bar{q}_{i+1}^{\prime \prime \prime}\left\lceil k \Vdash C\left(\bar{q}_{i+1}^{\prime \prime \prime}(k)\right) \cap\right.$ $\left(\bar{\alpha}_{i}^{\prime}, \bar{\alpha}_{i+1}+1\right)=\emptyset$. Finally, take $\bar{q}_{i+1} \leq_{i, \bar{T}_{i}} \bar{q}_{i+1}^{\prime \prime \prime}$ which is $\left(\bar{T}_{i+1}, i+1\right)-$ determined. We can find such a $\bar{q}_{i+1}$ by an argument similar to the one in Lemma 4.7. Then $\bar{q}_{i+1}$ satisfies (i), (ii), and (iii). This finishes the proof of Claim 4.9.1.

Given Claim 4.9.1, construct a sequence $\left\langle\bar{q}_{i}: i\langle\kappa\rangle\right.$ as follows: Take $\bar{q}_{1}$, $\bar{T}_{1}$ as already constructed. Given $\bar{q}_{i}, \bar{T}_{i}$, let $\bar{T}_{i+1} \supseteq \bar{T}_{i} \cup \bar{S}_{i+1} \cup$ (some part of $\left.\operatorname{supp}\left(\bar{q}_{i}\right)\right)$ be such that $\left|\bar{T}_{i+1}\right|<\kappa$, and take $\bar{q}_{i+1}$ satisfying Claim 4.9.1. For limit $i<\kappa$, let $\bar{q}_{i}=\bigcap_{i^{\prime}<i} \bar{q}_{i^{\prime}}$ and $\bar{T}_{i}=\bigcup_{i^{\prime}<i} \bar{T}_{i^{\prime}}$. Finally, let $\bar{r}=\bigcap_{i<\kappa} \bar{q}_{i}$. Choose the $\bar{T}_{i+1}$ 's so that $\bigcup_{i<\kappa} \bar{T}_{i}=\operatorname{supp}(\bar{r})$.

Now let $\left\langle S_{i}: i<j(\kappa)\right\rangle,\left\langle T_{i}: i<j(\kappa)\right\rangle$, and $\left\langle\alpha_{i}: i<j(\kappa)\right\rangle$ be the results of applying $j^{*}$ to $\left\langle\bar{S}_{i}: i<\kappa\right\rangle,\left\langle\bar{T}_{i}: i<\kappa\right\rangle$, and $\left\langle\bar{\alpha}_{i}: i<\kappa\right\rangle$, respectively. Note that $T_{\kappa+1} \supseteq S_{\kappa+1} \supseteq S$ and $\alpha_{\kappa+1} \geq \alpha$.

Let $r$ denote $j^{*}(\bar{r})$. Since (i) holds for $\bar{r}$ for all $\bar{i}<\kappa$, (i) holds for $r$ for all $i<j(\kappa)$, by elementarity. In particular, $r$ is $\left(T_{\kappa+1}, \kappa+1\right)$-determined with heights of length $\alpha^{\prime}$ for some ordinal $\alpha^{\prime} \geq \alpha_{\kappa+1} \geq \alpha+1$. (ii) holds for $r$ for all $i<j(\kappa)$. In particular, for each $k \in T_{\kappa+1} \backslash T_{\kappa}$, for each $\vec{x} \in\left(2^{\alpha^{\prime}}\right)^{T_{\kappa+1}}$ which lies on $r, r(\vec{x})\left\lceil k \Vdash \operatorname{ht}(\operatorname{stem}(r(\vec{x})(k))) \geq \alpha_{\kappa+1}+1\right.$. (iii) holds for $r$ for all $i<j(\kappa)$. In particular, for each $k \in T_{\kappa}$, for each $\vec{x} \in\left(2^{\alpha^{\prime}}\right)^{T_{\kappa+1}}$ which lies on $r, r(\vec{x})\left\lceil k \Vdash C(r(\vec{x})(k)) \backslash\left(\alpha_{\kappa}+1\right) \subseteq j^{*}(C)\right.$.

Since $T_{\kappa}$ is just $j\left[\bar{T}_{\kappa}\right]$, which equals $T_{\kappa+1} \cap \operatorname{ran}(j), r$ satisfies (1)-(3) of the lemma.

Now given our $\bar{q} \in g$, by the above argument, there is an $\bar{r} \in g$ below $\bar{q}$ such that $r=j^{*}(\bar{r})$ satisfies (1)-(3) of the lemma. As $T_{\kappa}$ has size $\kappa$, it follows that $M^{*}$ does contain the sequence $\left\langle\bar{x}(k): k \in T_{\kappa}\right\rangle$, by Lemma 4.4, and therefore, the sequence $\left\langle\bar{x}\left(j^{-1}(k)\right): k \in T_{\kappa}\right\rangle$. So the sequence of conditions $\left\langle r_{k}: k \in T_{\kappa}\right\rangle$ does belong to $M^{*}$, and we can form the condition $r^{*}=r \cap\left\{r_{k}\right.$ : $\left.k \in T_{\kappa}\right\}$ in $M^{*}$.

Let $\left\langle k_{l}: l<\right.$ o.t. $\left.\left(T_{\kappa+1}\right)\right\rangle$ enumerate $T_{\kappa+1}$ as a strictly increasing sequence. Proceed by induction on $l<$ o.t. $\left(T_{\kappa+1}\right) . r^{*} \uparrow k_{l}$ computes $r\left(k_{l}\right)$ up to height $\alpha+1$ as a ground model object. If $k_{l} \in T_{\kappa}$, then $\bar{x}\left(j^{-1}\left(k_{l}\right)\right) \frown 0$ is a branch in $r\left(k_{l}\right)$ up to height $\kappa+1$. In the interval $(\kappa+1, \alpha+1), r\left(k_{l}\right)$ does not split; so there is a unique branch in $r\left(k_{l}\right)$ of height $\alpha+1$ extending $\bar{x}\left(j^{-1}\left(k_{l}\right)\right) \frown 0$. If 
$k_{l} \in T_{\kappa+1} \backslash T_{\kappa}$, then $r\left\lceil k_{l}\right.$ forces $r\left(k_{l}\right) \cap 2^{\alpha+1}$ to be a ground model object which has no splitting below level $\alpha+1$. Let $y\left(k_{l}\right)$ be what $r\left\lceil k_{l}\right.$ forces $r\left(k_{l}\right) \cap 2^{\alpha+1}$ to be. Note that $r^{*}\left\lceil k_{l} \Vdash y\left(k_{l}\right) \subseteq \operatorname{stem}\left(r^{*}\left(k_{l}\right)\right)\right.$. This concludes the proof of Lemma 4.9.

Using $r^{*}$ from Lemma 4.9, note that $r^{*}$ is an $(S, i)$-thinning of $q$ by the argument in the previous paragraph, since $T_{\kappa+1} \supseteq S$. Since $q$ is $(S, i)$ determined, we obtain a condition below $q$ which is in $D \cap h$. Thus, $h$ is $\mathbb{Q}_{j(\kappa)}^{*}$ generic over $M^{*}$. This concludes the proof of the Main Claim.

This concludes the proof of Theorem 4.1.

\section{A lower bound on the consistency strength of the tree prop-} erty at the double successor of a measurable. Silver showed that if a regular cardinal $\kappa$ has the tree property in $V$ then it also has the tree property in $L$ [25]. He did this by associating to each $\kappa$-tree $T$ in $L$ another $\kappa$-tree $T^{*}$ in $L$ which has a $\kappa$-branch in $V$ iff $T$ has a $\kappa$-branch in $L$. (See [12] for a proof.) We generalize Silver's result as follows.

Lemma 5.1. Suppose that 0-pistol does not exist and let $K$ denote the core model. If an ordinal $\kappa$ of uncountable cofinality is inaccessible in $K$ and $T \in K$ is a $\kappa$-tree, then there is another $\kappa$-tree $T^{*}$ in $K$ such that $T^{*}$ has a $\kappa$-branch in $V$ iff $T$ has a $\kappa$-branch in $K$.

Proof. A node of $T^{*}$ consists of a triple $(M, \alpha, b)$ in $K$ such that $M$ is a mouse which agrees with $K$ below $\alpha<\kappa, b \in M$ is a branch through $T$ of length at least $\alpha$ and $M$ is the $\Sigma_{1}$-Skolem hull of $\alpha \cup\{b\}$. We say that a node $\left(M_{1}, \alpha_{1}, b_{1}\right)$ extends another node $\left(M_{0}, \alpha_{0}, b_{0}\right)$ of $T^{*}$ iff there is a (unique) $\Sigma_{1}$-elementary embedding of $M_{0}$ into $M_{1}$ which is the identity on $\alpha_{0}$ and sends $b_{0}$ to $b_{1}$.

$T^{*}$ has nodes of arbitrarily large rank less than $\kappa$. Indeed, let $\gamma$ be any successor cardinal of $K$ less than $\kappa$, let $b$ be a branch through $T$ of length $\gamma$ and for $\alpha<\gamma$ let $H_{\alpha}$ be the $\Sigma_{1}$-Skolem hull of $\alpha \cup\{b\}$ in $K$. Then for club-many $\alpha<\gamma, H_{\alpha} \cap \gamma=\alpha$ and the triple $\left(\bar{H}_{\alpha}, \alpha, \bar{b}_{\alpha}\right)$ is a node of $T^{*}$, where $\pi_{\alpha}: H_{\alpha} \simeq \bar{H}_{\alpha}$ is the transitive collapse and $\bar{b}_{\alpha}=\pi_{\alpha}(b)$. And for any two such $\alpha_{0}<\alpha_{1}$, the node $\left(\bar{H}_{\alpha_{1}}, \alpha_{1}, \bar{b}_{\alpha_{1}}\right)$ extends the node $\left(\bar{H}_{\alpha_{0}}, \alpha_{0}, \bar{b}_{\alpha_{0}}\right)$ in $T^{*}$. Similarly, any node $(M, \alpha, b)$ of $T^{*}$ with $\alpha$ at least $\gamma$ has rank at least $\gamma$ in $T^{*}$. It follows that $T^{*}$ is a $\kappa$-tree, as for any $\alpha$ less than $\kappa$ there are at most $\left(\alpha^{+}\right)^{K}<\kappa$ possibilities for a node of the form $(M, \alpha, b)$.

Suppose that $T$ has a $\kappa$-branch $b$ in $K$. Then as above it is easy to construct a $\kappa$-branch through $T^{*}$, by considering the $\Sigma_{1}$-Skolem hull of $\alpha \cup\{b\}$ in $K$ for $\alpha<\kappa$. Conversely, suppose that $T^{*}$ has a $\kappa$-branch in $V$. The direct limit along this branch is well-founded as $\kappa$ has uncountable cofinality. Let $(M, \kappa, b)$ be the direct limit along this branch, and note that $M$ is a mouse 
as it is $\Sigma_{1}$-projectible to $\kappa$ and therefore any potential counterexample to iterability would appear in one of the mice appearing along the $\kappa$-branch through $T^{*}$ leading to $M$. So $M$ is a mouse $\Sigma_{1}$-projecting to $\kappa$ which agrees with $K$ below $\kappa$. It follows that $M$ agrees with $K$ below $\left(\kappa^{+}\right)^{M}$, as $M$ agrees with its core below this ordinal, and the core of $M$ is an initial segment of $K$. As $b\left\lceil\kappa\right.$ belongs to $M$ and therefore to $M \uparrow\left(\kappa^{+}\right)^{M}$ and the latter is an element of $K$, we obtain the $\kappa$-branch $b\lceil\kappa$ through $T$ in $K$, as desired.

We thank Martin Zeman for helpful discussions regarding Lemma 5.1.

Corollary 5.2. Suppose that 0-pistol does not exist and let $K$ be the core model. Then for any regular cardinal $\kappa$, if $\kappa$ has the tree property in $V$ then $\kappa$ is weakly compact in $K$.

Proof. Suppose that $\kappa$ has the tree property in $V$. Then $\kappa$ is inaccessible in $K$ : Otherwise, let $\gamma^{K}$ be the largest $K$-cardinal less than $\kappa$ and $\gamma$ be the largest cardinal less than $\kappa$. As $\square_{\gamma^{K}}$ holds in $K$, it follows that $\square_{\gamma}$ holds in $V$. But $\square_{\gamma}$ implies the existence of a $\kappa$-Aronszajn tree (by [27]), contradicting the tree property at $\kappa$.

Now Lemma 5.1 implies that $\kappa$ has the tree property in $K$. As $\kappa$ is inaccessible in $K$, it follows that $\kappa$ is weakly compact in $K$, as for inaccessible cardinals, the tree property is equivalent to weak compactness.

See Schindler 24] for an alternative proof of a slightly weaker version of Corollary 5.2 ,

TheOREM 5.3. If TP( $\left.\kappa^{++}\right)$holds where $\kappa$ is measurable, then there is an inner model in which $o^{K}(\kappa) \geq \lambda+1$, where $\lambda>\kappa$ is weakly compact in that inner model.

Proof. If 0-pistol exists, then by iterating 0-pistol through the ordinals, we obtain an inner model with a strong cardinal together with a proper class of weakly compact cardinals. In particular, this strong cardinal is weakly compact hypermeasurable. So assume that 0-pistol does not exist. In this case, the core model $K$ exists. By Theorem 1.4 of [11, o ${ }^{K}(\kappa) \geq \kappa^{++}$. By Corollary $5.2, \kappa^{++}$is weakly compact in $K$. Therefore, $\mathrm{o}^{K}(\kappa) \geq \lambda$ for $\lambda$ a weakly compact cardinal in $K$ above $\kappa$. Hence, in every case, we obtain the consistency of a measurable cardinal $\kappa$ with $\mathrm{o}^{K}(\kappa)$ at least the next weakly compact above $\kappa$.

We claim that $o^{K}(\kappa)$ cannot be a weakly compact cardinal. Let $\lambda$ be a weakly compact cardinal above $\kappa$. Then $\lambda$ is $\Pi_{1}^{1}$-reflecting. Suppose $\kappa$ is $<\lambda$ weakly compact hypermeasurable (i.e. for each cardinal $\theta<\lambda, \kappa$ is $\theta$-hypermeasurable). " $\kappa$ is $\theta$-hypermeasurable" is a $\Sigma_{1}^{1}$-property about $H(\theta)$. Hence, by reflection, $\kappa$ is $\lambda$-hypermeasurable. Thus, actually o ${ }^{K}(\kappa) \geq \lambda+1$, where $\lambda$ is the least weakly compact above $\kappa$. 
We thank Joel Hamkins for pointing out the reflection argument in Theorem 5.3 .

Theorem 4.1 of Section 4 gives the upper bound for the tree property at the double successor of a measurable cardinal. Theorem 5.3 of Section 5 provides the lower bound. Hence, we have the Main Theorem.

Main Theorem. " $\kappa$ is a measurable cardinal and $T P\left(\kappa^{++}\right)$holds" is equiconsistent over ZFC with " $\kappa$ is weakly compact hypermeasurable."

Note: By Lemma 4.4 and the remarks immediately following, the weakly compact hypermeasurable remains $H\left(\kappa^{++}\right)$-hypermeasurable in the generic extension. (A cardinal $\kappa$ is $H\left(\kappa^{++}\right)$-hypermeasurable if there is an elementary embedding $j: V \rightarrow M$ such that $\operatorname{crit}(j)=\kappa$, and the $H\left(\kappa^{++}\right)$of $V$ and $M$ are the same.) In fact, every weakly compact hypermeasurable less than or equal to $\kappa$ will have its $H\left(\kappa^{++}\right)$-hypermeasurability preserved.

\section{Internal consistency}

TheOREM 6.1. Suppose $V \models G C H$ and has a weakly compact hypermeasurable cardinal $\kappa$ which is a limit of weakly compact hypermeasurable cardinals, together with a measurable cardinal $\mu$ above $\kappa$. Then there is an inner model of $V$ in which there is a proper class of $H\left(\rho^{++}\right)$-hypermeasurable cardinals $\rho$, and in which the tree property holds at the double successor of each strongly inaccessible cardinal.

Proof. The proof is similar to that of Theorem 3.2 in [4]. Let $U$ be a normal measure on $\mu$ and let $\pi: M \rightarrow H(\theta)$ be the inverse of the transitive collapse of a countable elementary submodel of some large $H(\theta)$, with $\kappa, \mu$ and $U$ in the range of $\pi$. Let $\bar{\kappa}, \bar{\mu}, \bar{U}$ be the preimages under $\pi$ of $\kappa, \mu$, and $U$, respectively.

Note that $\bar{U}$ is an iterable measure, as $U$ is iterable. Now choose a generic $G$ over $M$ for the reverse Easton iteration of length $\bar{\kappa}+1$ of the sums $\bigoplus\left\{\operatorname{Sacks}\left(\rho_{i}, \lambda\right): \lambda \in\left(\rho_{i}, \lambda_{i}\right]\right.$ and $\lambda$ is strongly inaccessible $\}(i \leq \bar{\kappa})$ as in the Main Theorem. Such a $G$ exists by the countability of $M$. Then $\bar{U}$ lifts to an iterable measure $(\bar{U})^{*}$ in $M[G]$ as the forcing to add $G$ has size less than $\bar{\mu}$, and all weakly compact hypermeasurables $\bar{\rho}$ of $M$ (including $\bar{\kappa}$ ) remain $H\left(\bar{\rho}^{++}\right)$-hypermeasurable in $M[G]$. Iterate $M[G]$ via $(\bar{U})^{*}$ through the ordinals, resulting in an inner model $N^{\prime}$.

Then $\bar{\kappa}$ is measurable in $N^{\prime}$, and any normal measure $W$ on $\bar{\kappa}$ in $N^{\prime}$ is iterable as $N^{\prime}$ contains all the ordinals. Now iterate $H\left(\bar{\kappa}^{+}\right)$of $N^{\prime}$ via some $W$ through the ordinals, producing the elementary chain $\left\langle H\left(\bar{\kappa}_{i}\right)^{N_{i}^{\prime}}: i<\right.$ Ord $\rangle$. Let $N$ be the inner model $\bigcup_{i \in \text { Ord }} H\left(\bar{\kappa}_{i}\right)^{N_{i}^{\prime}}$. Then in $N$ there is a proper class of cardinals $\rho$ which are $H\left(\rho^{++}\right)$-hypermeasurable and the tree property holds at the double successor of all strongly inaccessible cardinals. 
Theorem 6.2. Suppose $0^{\#}$ exists. Then there is an inner model in which the tree property holds at the double successor of every strongly inaccessible cardinal.

Proof. The proof follows by combining our methods from Section 4 with the template provided by Friedman and Thompson in [9] for constructing a class generic using $0^{\#}$ and some results from [10. We only need to check that each stage of our proof in Section 4 can be transferred to the $0^{\#}$ setting with no glitches.

Let $\rho_{0}$ be the least strongly inaccessible in $L$ and let $\lambda_{0}$ be the least weakly compact above $\rho_{0}$ in $L$. Given $\lambda_{i}$, let $\rho_{i+1}$ be the least strongly inaccessible in $L$ above $\lambda_{i}$ and let $\lambda_{i+1}$ be the least weakly compact in $L$ above $\rho_{i+1}$. For limit ordinals $i$, let $\rho_{i}$ be the least strongly inaccessible in $L$ greater than or equal to $\sup _{k<i} \rho_{k}$, and let $\lambda_{i}$ be the least weakly compact greater than $\rho_{i}$ in $L$. Let $\mathbb{P}$ be the class forcing defined in $L$ with reverse Easton support obtained by the iteration $\left\langle\left\langle\mathbb{P}_{i}, \dot{\mathbb{Q}}_{i}\right\rangle: i \in \operatorname{Ord}\right\rangle$, where $\mathbb{P}_{0}$ is the trivial forcing and for each ordinal $i, \dot{\mathbb{Q}}_{i}$ is a $\mathbb{P}_{i}$ name for $\operatorname{Sacks}\left(\rho_{k}, \lambda_{k}\right)$ if $i=\rho_{k}$ for some $k ; \dot{\mathbb{Q}}_{i}$ is a name for the trivial forcing otherwise. Since the elementary embeddings will be from $L$ into $L$, we do not need to use sums as the iterands of $\mathbb{P}$.

List all the indiscernibles as $I=\left\langle i_{\beta}: \beta \in\right.$ Ord $\rangle$. Note that for each $\beta \in$ Ord, $i_{\beta}$ is in $L$ a strongly inaccessible limit of weakly compacts, so $\rho_{i_{\beta}}=i_{\beta}$ and $\dot{\mathbb{Q}}_{i_{\beta}}$ is a $\mathbb{P}_{i_{\beta}}$ name for $\operatorname{Sacks}\left(\rho_{i_{\beta}}, \lambda_{i_{\beta}}\right)$. For each $i_{\beta}<i_{\gamma}$ indiscernibles, let $\pi_{i_{\beta}, i_{\gamma}}$ denote the elementary embedding from $L$ to $L$ which fixes all indiscernibles below $i_{\beta}$, and moves each indiscernible $i_{\varepsilon} \geq i_{\beta}$ to $i_{\gamma+(\varepsilon-\beta)}$.

Let $G\left(<i_{\beta}\right)$ denote the generic for $\mathbb{P}_{i_{\beta}}$ and $G\left(\leq i_{\beta}\right)$ denote the generic for $\mathbb{P}_{i_{\beta}+1}$. By work in [9], if we can satisfy the following induction hypothesis at every stage, then the class generic can be built:

Induction Hypothesis.

$$
\forall i_{\beta}, i_{\gamma} \in I, i_{\beta}<i_{\gamma} \rightarrow \pi_{i_{\beta}, i_{\gamma}}\left[G\left(\leq i_{\beta}\right)\right] \subseteq G\left(\leq i_{\gamma}\right) .
$$

Lemma 4.3 in [9] (see also the first appearance of this argument in [8]) and $(*)$ ensure that we can build the generic at each limit indiscernible $i_{\beta}$, provided that we have the generic for each indiscernible less than $i_{\beta}$. For $i_{\gamma}$ a limit indiscernible, we will have $G\left(\leq i_{\gamma}\right)=\bigcup_{\beta<\gamma} \pi_{i_{\gamma}, i_{\beta}}\left[G\left(\leq i_{\gamma}\right)\right]$ generic for $\mathbb{P}\left(\leq i_{\gamma}\right)$. Thus, we only need to consider the successor case: given $G\left(\leq i_{\beta}\right)$ generic for $P_{i_{\beta+1}}$, find a generic $G\left(\leq i_{\beta+1}\right)$ for $P\left(\leq i_{\beta+1}\right)$ such that $(*)$ holds. Building the generic $G\left(i_{\beta}, i_{\beta+1}\right)$ for $\mathbb{P}\left\lceil\left(i_{\beta}, i_{\beta+1}\right)\right.$ over $L\left[G\left(\leq i_{\beta}\right)\right]$ can be achieved, using Lemma 4.4 in [9]. Also, since $i_{0}$ is countable in $V$, the base case of the construction of the generic is sound. 
Now construct $G\left(i_{\beta+1}\right)$. Repeat the argument of Section 4 changing only the following few details. Let $G\left(i_{\beta+1}\right)$ be the filter generated in $L\left[G\left(<i_{\beta+1}\right)\right]$ by $\pi_{i_{\beta}, i_{\beta+1}}\left[G\left(i_{\beta}\right)\right] \cup\left\{\bigcap_{i \in I} r_{i}: I \in L\left[G\left(<i_{\beta+1}\right)\right], I \subseteq \lambda_{i_{\beta+1}} \cap \operatorname{ran}\left(\pi_{i_{\beta}, i_{\beta+1}}\right)\right.$, and $\left.|I| \leq \rho_{i_{\beta+1}}\right\}$, where $r_{i}$ is defined in $L\left[G\left(<i_{\beta}\right)\right]$ analogously as in Theorem 4.1.

Definition 4.6, Lemma 4.7, and Definition 4.8 do not need to be changed. For the analogue of Lemma 4.9, note that in this setting, each dense subset of $\operatorname{Sacks}\left(\rho_{i_{\beta+1}}, \lambda_{i_{\beta+1}}\right)$ in $L\left[G\left(<i_{\beta+1}\right)\right]$ can be written as $\pi_{i_{\beta}, i_{\beta+1}}^{*}(f)\left(i_{\beta+1}\right)$, where $\pi_{i_{\beta}, i_{\beta+1}}^{*}$ is the lifting of $\pi_{i_{\beta}, i_{\beta+1}}$ to $\pi_{i_{\beta}, i_{\beta+1}}^{*}: L\left[G\left(<i_{\beta}\right)\right] \rightarrow L\left[G\left(<i_{\beta+1}\right)\right]$, $f \in L\left[G\left(<i_{\beta+1}\right)\right]$, and $\operatorname{dom}(f)=i_{\beta}$. Given the $\alpha$ corresponding to the $(S, i)$ for which we need to find an $(S, i)$-thinning of $q$ in $G\left(i_{\beta+1}\right)$, use a club set $C \subseteq i_{\beta}$ in $L$ such that $\pi_{i_{\beta}, i_{\beta+1}}(C) \cap\left(i_{\beta}, \alpha\right)=\emptyset$. Lemma 11 in [10] ensures that the condition $r^{*}$ in our Lemma 4.9 really is in $L\left[G\left(<i_{\beta+1}\right)\right]$. The rest follows as in Section 4.

7. Special Aronszajn trees. In this section, we apply our proof methods to the case of special Aronszajn trees, which was kindly suggested to us by Ali Enayat.

Definition $7.1([16])$. A $\rho^{++}$-tree $T$ is a special Aronszajn tree if $T$ is a subset of $\left\{f: f\right.$ is a 1-1 function from an ordinal less than $\rho^{++}$into $\left.\rho^{+}\right\}$ closed under initial segments such that for each $\alpha<\rho^{++}, T$ has at most $\rho^{+}$ elements with domain $\alpha$.

The following is stated in Kanamori [15].

TheOREM 7.2. Assume GCH holds in $V$. Let $\rho<\lambda$, where $\rho$ either is $\aleph_{0}$ or is strongly inaccessible and $\lambda$ is Mahlo in $V$. Then $\operatorname{Sacks}(\rho, \lambda)$ produces a generic extension $V[G]$ in which there is no special $\rho^{++}$-Aronszajn tree.

Proof. Let $G$ be generic for $\operatorname{Sacks}(\rho, \lambda)$ over $V$. Then $\operatorname{Sacks}(\rho, \lambda)$ preserves $\rho^{+}$, collapses $\lambda$ to $\left(\rho^{++}\right)^{V[G]}$, and adds $\left(\rho^{++}\right)^{V[G]}$ many new subsets of $\rho$. Suppose that $T=\dot{T}^{G}$ were a special $\rho^{++}$-Aronszajn tree in $V[G]$. Then since $\lambda$ is Mahlo in $V$, we can choose an inaccessible $\alpha<\lambda$ in $V$ so that $T\left\lceil\alpha \in V\left[G\lceil\alpha]\right.\right.$ and is a special Aronszajn tree in $V\left[G\lceil\alpha]\right.$. As $\alpha$ is $\rho^{++}$in $V[G\lceil\alpha]$, it follows that $T\lceil\alpha$ has no cofinal branch in $V[G\lceil\alpha]$. However, $T\lceil\alpha$ does have a cofinal branch in $V[G]$, since $T$ has nodes on level $\alpha$. Let $\dot{b}$ be a $\operatorname{Sacks}(\rho, \lambda)$ name for a cofinal branch through $T\lceil\alpha$. As in the case of (general) Aronszajn trees, build a perfect tree $S$ of conditions in $\operatorname{Sacks}(\rho, \lambda) \uparrow[\alpha, \lambda)$ of height $\rho$ such that any infinite branch through $S$ is a fusion sequence with a lower bound, and such that distinct infinite branches through $S$ force different facts about $\dot{b}$, and hence, produce distinct branches through $T\lceil\alpha$, of some height $\beta<\alpha$. But then in $V\left[G\lceil\alpha], T\left\lceil\alpha\right.\right.$ has $\left(2^{\rho}\right)^{V[G\lceil\alpha]}=\alpha$ many nodes on level $\beta$. This contradicts the fact that $T\lceil\alpha$ is a special Aronszajn tree in $V[G\lceil\alpha]$. 
We say that $\kappa$ is Mahlo hypermeasurable if there is a Mahlo cardinal $\lambda>\kappa$ and an elementary embedding $j: V \rightarrow M$ such that $\kappa=\operatorname{crit}(j)$ and $(H(\lambda))^{V}=(H(\lambda))^{M}$. Hence, if $\kappa$ is Mahlo hypermeasurable, then there is a forcing extension where $\kappa$ is measurable and there is no special Aronszajn tree on $\kappa^{++}$. We say that $\kappa$ is $<$ Mahlo hypermeasurable if for any $\alpha$ less than some Mahlo above $\kappa, \kappa$ is $\alpha$-hypermeasurable. Conversely, $\operatorname{Con}(\kappa$ is measurable and there is no special Aronszajn tree on $\left.\kappa^{++}\right)$implies Con $(\kappa$ is $<$ Mahlo hypermeasurable), by work of Gitik [11], as either 0-pistol exists, or $K$ exists and $o^{K}(\kappa)$ is Mahlo in $K$.

Thus, we have the following theorem.

THEOREM 7.3. An upper bound for the consistency strength of the tree property for special Aronszajn trees at the double successor of a measurable cardinal is a Mahlo hypermeasurable. A lower bound for the consistency strength of the tree property for special Aronszajn trees at the double successor of a measurable cardinal is at least a $<$ Mahlo hypermeasurable.

Since Mahlo cardinals are downwards absolute, we can just use the appropriate $\operatorname{Sacks}\left(\rho_{i}, \lambda_{i}\right)$ for the iterands in the forcing for the proof of the previous theorem instead of sums of such Sacks iterations.

We conclude this paper with the following open problems.

Open Problem 7.4. Find the exact consistency strength of the special tree property at the double successor of a measurable cardinal.

Open Problem 7.5. Is it consistent with ZFC that there is a measurable cardinal $\kappa$, the tree property holds at $\kappa^{++}$, and there is a definable well-ordering of $H\left(\kappa^{++}\right)$?

Open Problem 7.6. Is it consistent with ZFC that the tree property holds simultaneously at all even successor cardinals?

Open Problem 7.7. Assume $0^{\#}$. Let $f$ be an Easton function defined on the regular cardinals in $L$ such that $f$ is $L$-definable without parameters. Is there an inner model realizing $f$ such that the tree property holds at the double successor of each inaccessible?

Acknowledgements. The first author would like to thank FWF grant P16790 - N04 for support of this research.

The second author would like to thank FWF grants P16790 - N04 and P19375 - N18 for support of this research.

\section{References}

[1] U. Abraham, Aronszajn trees on $\aleph_{2}$ and $\aleph_{3}$, Ann. Pure Appl. Logic 24 (1983), 213-230. 
[2] J. Baumgartner and R. Laver, Iterated perfect set forcing, Ann. Math. Logic 17 (1979), 271-288.

[3] J. Cummings and M. Foreman, The tree property, Adv. Math. 133 (1998), 1-32.

[4] N. Dobrinen and S.-D. Friedman, Internal consistency and global co-stationarity of the ground model, J. Symbolic Logic 73 (2008), 512-521.

[5] M. Foreman, M. Magidor, and R.-D. Schindler, The consistency strength of successive cardinals with the tree property, ibid. 66 (2001), 1837- 1847.

[6] S.-D. Friedman, Fine Structure and Class Forcing, de Gruyter, 2000.

[7] S.-D. Friedman and R. Honzík, Easton's theorem and large cardinals, Ann. Pure Appl. Logic 154 (2008), 191-208.

[8] S.-D. Friedman and P. Ondrejovič, The internal consistency of Easton's theorem, ibid. 156 (2008), 259-269.

[9] S.-D. Friedman and K. Thompson, Internal consistency for embedding complexity, J. Symbolic Logic 73 (2008), 831-844.

[10] -, -, Perfect trees and elementary embeddings, ibid. 73 (2008), 906-918.

[11] M. Gitik, On measurable cardinals violating the continuum hypothesis, Ann. Pure Appl. Logic 63 (1993), 227-240.

[12] L. Harrington and S. Shelah, Some exact equiconsistency results in set theory, Notre Dame J. Formal Logic 26 (1985), 178-188.

[13] T. Jech, Set Theory. The Third Millennium Edition, Revised and Expanded, Springer, 2003.

[14] R. B. Jensen, The fine structure of the constructible hierarchy, Ann. Math. Logic 4 (1972), 229-308.

[15] A. Kanamori, Perfect-set forcing for uncountable cardinals, ibid. 19 (1980), 97-114.

[16] —, The Higher Infinite, 2nd ed., Springer, 2003.

[17] D. König, Über eine Schlussweise aus dem Endlichen ins Unendliche: Punktmengen. Kartenfärben. Verwandtschaftsbeziehungen. Schachspiel, Acta Sci. Math. 3 (1927), 121-130.

[18] D. Kurepa, Ensembles ordonnés et ramifiés, Publ. Math. Univ. Belgrade 4 (1935), $1-138$.

[19] R. Laver, Making the supercompactness of $\kappa$ indestructible under $\kappa$-directed closed forcing, Israel J. Math. 29 (1978), 385-388.

[20] M. Magidor and S. Shelah, The tree property at successors of singular cardinals, Arch. Math. Logic 35 (1996), 385-404.

[21] W. Mitchell, Aronszajn trees and the independence of the transfer property, Ann. Math. Logic 5 (1972/73), 21-46.

[22] T. Miyamoto, unpublished notes.

[23] G. E. Sacks, Forcing with perfect closed sets, in: Axiomatic Set Theory (Los Angeles, CA, 1967), Part I, Proc. Sympos. Pure Math. 13, Amer. Math. Soc., 1971, 331-355.

[24] R. Schindler, Weak covering and the tree property, Arch. Math. Logic 38 (1999), 515-520.

[25] J. Silver, Some applications of model theory in set theory, Ann. Math. Logic 3 (1971), 45-110.

[26] E. Specker, Sur un problème de Sikorski, Colloq. Math. 2 (1949), 9-12. 
[27] S. Todorčević, Aronszajn trees and partitions, Israel J. Math. 52 (1985), 53-58.

Natasha Dobrinen

Sy-David Friedman

Department of Mathematics

University of Denver

Kurt Gödel Research Center for Mathematical Logic

2360 S Gaylord St

Universität Wien

Denver, CO 80208, U.S.A.

E-mail: natasha.dobrinen@du.edu

Währinger Strasse 25

http://www.math.du.edu/dobrinen/

A-1090 Wien, Austria

E-mail: sdf@logic.univie.ac.at http://www.logic.univie.ac.at/ sdf

Received 13 November 2008;

in revised form 28 December 2009 